Article

\title{
Investigation of Ulam Stability Results of a Coupled System of Nonlinear Implicit Fractional Differential Equations
}

\author{
Zeeshan Ali ${ }^{1}{ }^{1}$, Poom Kumam ${ }^{2,3, *} \mathbb{C}$, Kamal Shah ${ }^{4} \mathbb{D}$ and Akbar Zada ${ }^{1} \mathbb{C}$ \\ 1 Department of Mathematics, University of Peshawar, Peshawar, Khyber Pakhtunkhwa 25000, Pakistan; \\ zeeshanmaths1@gmail.com (Z.A.); akbarzada@uop.edu.pk (A.Z.) \\ 2 Center of Excellence in Theoretical and Computational Science (TaCS-CoE) \& KMUTTFixed Point Research \\ Laboratory, Room SCL 802 Fixed Point Laboratory, Science Laboratory Building, Departments of \\ Mathematics, Faculty of Science, King Mongkut's University of Technology Thonburi (KMUTT), 126 \\ Pracha-Uthit Road, Bang Mod, Thrung Khru, Bangkok 10140, Thailand \\ 3 Department of Medical Research, China Medical University Hospital, China Medical University, \\ Taichung 40402, Taiwan \\ 4 Department of Mathematics, University of Malakand, Dir(L), Khyber Pakhtunkhwa 18800, Pakistan; \\ kamal@uom.edu.pk \\ * Correspondence: kumampoom@gmail.com or Poom.kum@kmutt.ac.th
}

Received: 18 February 2019; Accepted: 28 March 2019; Published: 9 April 2019

\begin{abstract}
This manuscript deals with the existence theory, uniqueness, and various kinds of Ulam-Hyers stability of solutions for a class and coupled system of fractional order differential equations involving Caputo derivatives. Applying Schaefer and Banach's fixed point approaches, existence and uniqueness results are obtained for the proposed problems. Stability results are investigated by using the classical technique of nonlinear functional analysis. Examples are given with each problem to illustrate the main results.
\end{abstract}

Keywords: Caputo fractional derivative; implicit differential equation; Green's function; implicit coupled system; existence theory; Ulam stability

MSC: 26A33; 34B27; 39B82; 45M10

\section{Introduction}

Fractional order differential equations (FODE) are generalizations of ordinary differential equations to an arbitrary order. The aforesaid equations have obtained considerable attention from researchers because of their ability to model different complex phenomena. The foregoing equations capture nonlocal relations in space and time with power-law memory kernels. Due to the widespread applications of FODE in science and engineering, research in this field has grown significantly all around the world. The aforesaid equations arise in many disciplines of science and engineering as the mathematical modeling of systems and processes in the fields of biophysics, blood flow phenomena, signal and image processing, polymer rheology, control theory, the electrodynamics of a complex medium, physics, aerodynamics, economics, chemistry, etc. For details, see [1-7] and the references cited therein. However, the theory of boundary value problems for nonlinear FODE remains within the initial stages, and plenty of aspects of this theory have to need to be explored.

The research area, that is most ideal within the field of FODE and has received extraordinary interest from researchers, is devoted to the existence theory of solutions. Several researchers have built up some attention-grabbing results of the existence of solutions to boundary value problems 
for FODE by using standard fixed point theorems. For a detailed study, see [8-10] and the references cited therein. However, the study of coupled systems of differential equations of different orders is also very significant because this kind of system appears in different problems of an applied nature; see [11-19] and the references cited therein. Ahmad and Nieto [20] studied the existence of the solution to the following nonlinear FODE involving the Caputo derivative of fractional order, by using the Leray-Schauder degree theory:

$$
\left\{\begin{array}{l}
{ }^{c} \mathrm{D}^{\mathrm{p}} \mathrm{u}(\mathrm{t})-\alpha(\mathrm{t}, \mathrm{u}(\mathrm{t}))=0 ; \mathrm{t} \in \mathscr{J}, \\
\left.\mathrm{u}(\mathrm{t})\right|_{\mathrm{t}=0}=-\left.\mathrm{u}(\mathrm{t})\right|_{\mathrm{t}=\mathrm{T}},\left.\mathrm{u}^{\prime}(\mathrm{t})\right|_{\mathrm{t}=0}=-\left.\mathrm{u}^{\prime}(\mathrm{t})\right|_{\mathrm{t}=\mathrm{T}}
\end{array}\right.
$$

where $1<\mathrm{p} \leq 2, \mathscr{J}=[0, \mathrm{~T}]$ with $\mathrm{T}>0$, and $\alpha: \mathscr{J} \times \mathscr{R} \rightarrow \mathscr{R}$ is continuous.

The implicit FODE represent a very important class of differential equations. This manuscript is motivated by the importance of implicit ordinary differential equations of the form:

$$
\alpha\left(\mathrm{t}, \mathrm{u}(\mathrm{t}), \mathrm{u}^{\prime}(\mathrm{t}), \ldots, \mathrm{u}^{(\mathrm{n}-1)}(\mathrm{t})\right)=0
$$

under different initial and boundary conditions. Implicit equations have been considered by many researchers; see [21-25] and the references cited therein. Many researchers have extended the above results to the foregoing equations by the use of different fixed point approaches. Recently, some existence results for an implicit FODE on compact intervals have been: Benchohra and Lazreg [26], who investigated the existence results for the following nonlinear implicit FODE involving the Caputo derivative of fractional order:

$$
\left\{\begin{array}{l}
{ }^{c} D^{\mathrm{P}} \mathrm{u}(\mathrm{t})-\alpha\left(\mathrm{t}, \mathrm{u}(\mathrm{t}),{ }^{c} \mathrm{D}^{\mathrm{P}} \mathrm{u}(\mathrm{t})\right)=0 ; \mathrm{t} \in \mathscr{J}, \\
\left.\mathrm{u}(\mathrm{t})\right|_{\mathrm{t}=0}=\mathrm{u}_{0},\left.\mathrm{u}(\mathrm{t})\right|_{\mathrm{t}=\mathrm{T}}=\mathrm{u}_{1},
\end{array}\right.
$$

where $1<\mathrm{p} \leq 2, \mathscr{J}=[0, \mathrm{~T}]$ with $\mathrm{T}>0, \mathrm{u}_{0}, \mathrm{u}_{1} \in \mathscr{R}$, and $\alpha: \mathscr{J} \times \mathscr{R} \times \mathscr{R} \rightarrow \mathscr{R}$ is continuous. For more examples, the reader may see $[27,28]$ and the references cited therein.

Another area of research, which has received considerable attention from researchers, is the Ulam-Hyers stability analysis of the differential equations and their different kinds. The aforesaid stability was first introduced by Ulam [29] in 1940. The problem posed by Ulam was the following: "Under what conditions does there exist an additive mapping near an approximately additive mapping?". A significant breakthrough came in 1941 when Hyers [30] gave an answer to Ulam's problem in the case of Banach spaces: Let $\mathscr{E}_{1}, \mathscr{E}_{2}$ be real Banach spaces and $\epsilon>0$. Then, for each mapping $\alpha: \mathscr{E}_{1} \rightarrow \mathscr{E}_{2}$ satisfying:

$$
\|\alpha(\mathrm{u}+\mathrm{y})-\alpha(\mathrm{u})-\alpha(\mathrm{y})\| \leq \epsilon
$$

for all $\mathrm{u}, \mathrm{y} \in \mathscr{E}_{1}$, there is a unique additive mapping $\chi: \mathscr{E}_{1} \rightarrow \mathscr{E}_{2}$ with:

$$
\|\alpha(\mathrm{u})-\chi(\mathrm{u})\| \leq \epsilon, \text { for all } \mathrm{u} \in \mathscr{E} 1 .
$$

Afterward, this type of stability was known as the Ulam-Hyers stability. In addition to the aforesaid investigations, many researchers have investigated the Ulam stability for differential equations of different orders; see [31-34] and the references cited therein. The mentioned stability analysis is extremely helpful in numerous applications, for example numerical analysis and optimization and so forth, where it is very difficult to find the exact solution of a nonlinear problem and to provide a bridge between numerical and analytical solutions. The aforementioned stabilities [35] for FODE are rather significant in practical problems, economics, biology, and numerical analysis. For examples, see [36-40] and the references cited therein. Furthermore, the fractional order system may have an additional attractive feature over the integer order system. Let us suppose the following 
example to show which one is more stable in the aforementioned (fractional order and integer order) systems.

Example. [41]

Example 1. We have the following two systems with initial condition $u(0)$ for $v \in(0,1)$,

$$
\begin{aligned}
\frac{d}{d t} u(t) & =v t^{v-1}, \\
{ }_{0}^{c} D_{t}^{p} u(t) & =v t^{v-1}, \quad 0<p<1 .
\end{aligned}
$$

Then, the analytical solutions of (1) and (2) are $t^{v}+u(0)$ and $\frac{v \Gamma(v) t^{v+p-1}}{\Gamma(v+p)}+u(0)$, respectively. Clearly, the integer order system (1) is unstable for $0<v<1$. However, the fractional dynamic system (2) is stable for each $0<v \leq 1-\mathrm{p}$. Therefore, the fractional order system may have better features than the integer order system.

Benchohra and Lazreg [42] investigated the existence and different kinds of Ulam-Hyers stability for the following nonlinear implicit FODE involving the Caputo derivative of fractional order:

$$
\left\{\begin{array}{l}
{ }^{c} \mathrm{D}^{\mathrm{p}} \mathrm{u}(\mathrm{t})-\alpha\left(\mathrm{t}, \mathrm{u}(\mathrm{t}),{ }^{c} \mathrm{D}^{\mathrm{p}} \mathrm{u}(\mathrm{t})\right)=0 ; \mathrm{t} \in \mathscr{J}, \\
\left.\mathrm{u}(\mathrm{t})\right|_{\mathrm{t}=0}=\mathrm{u}_{0},
\end{array}\right.
$$

where $1<\mathrm{p} \leq 2, \mathscr{J}=[0, \mathrm{~T}]$ with $\mathrm{T}>0, \mathrm{u}_{0} \in \mathscr{R}$, and $\alpha: \mathscr{J} \times \mathscr{R} \times \mathscr{R} \rightarrow \mathscr{R}$ is continuous.

Ali et al. [43] investigated existence theory and different kinds of Ulam-Hyers stability for the following nonlinear implicit FODE involving the Riemann-Liouville fractional order derivative:

$$
\left\{\begin{array}{l}
D^{\mathrm{p}} \mathrm{u}(\mathrm{t})-\alpha\left(\mathrm{t}, \mathrm{u}(\mathrm{t}), \mathrm{D}^{\mathrm{p}} \mathrm{u}(\mathrm{t})\right)=0 ; \mathrm{t} \in \mathscr{J}, \\
\left.\mathrm{D}^{\mathrm{p}-2} \mathrm{u}(\mathrm{t})\right|_{\mathrm{t}=0^{+}}=\left.\mathrm{aD}^{\mathrm{p}-2} \mathrm{u}(\mathrm{t})\right|_{\mathrm{t}=\mathrm{T}^{-}} \\
\left.\mathrm{D}^{\mathrm{p}-1} \mathrm{u}(\mathrm{t})\right|_{\mathrm{t}=0^{+}}=\left.\mathrm{bD}^{\mathrm{p}-1} \mathrm{u}(\mathrm{t})\right|_{\mathrm{t}=\mathrm{T}^{-}}
\end{array}\right.
$$

where $1<\mathrm{p} \leq 2$ and $\mathscr{J}=[0, \mathrm{~T}]$ with $\mathrm{T}>0, \mathrm{a}, \mathrm{b} \neq 1$, and $\alpha: \mathscr{J} \times \mathscr{R} \times \mathscr{R} \rightarrow \mathscr{R}$ is continuous.

Currently, mathematicians have devoted their work to the investigation of various kinds of Ulam-Hyers stability for coupled systems of FODE. For details, see [44-48]. Currently, to the best of our knowledge, very few papers can be found in the literature in which the authors studied the existence theory and different kinds of Ulam-Hyers stability for the aforesaid system of nonlinear implicit FODE. Ali et al. [49] investigated existence theory and different kinds of Ulam-Hyers stability for the following implicit coupled system involving the Caputo derivative of fractional order:

$$
\left\{\begin{array}{l}
{ }^{c} \mathrm{D}^{\mathrm{p}} \mathrm{u}(\mathrm{t})-\alpha\left(\mathrm{t}, \mathrm{y}(\mathrm{t}),{ }^{c} \mathrm{D}^{\mathrm{p}} \mathrm{u}(\mathrm{t})\right)=0 ; \mathrm{t} \in \mathscr{J} \\
{ }^{c} \mathrm{D}^{\mathrm{q}} \mathrm{y}(\mathrm{t})-\chi\left(\mathrm{t}, \mathrm{u}(\mathrm{t}),{ }^{c} \mathrm{D}^{\mathrm{q}} \mathrm{y}(\mathrm{t})\right)=0 ; \mathrm{t} \in \mathscr{J} \\
\left.\mathrm{u}^{\prime}(\mathrm{t})\right|_{\mathrm{t}=0}=\left.\mathrm{u}^{\prime \prime}(\mathrm{t})\right|_{\mathrm{t}=0}=0,\left.\mathrm{u}(\mathrm{t})\right|_{\mathrm{t}=1}=\tau \mathrm{u}(s) \\
\left.\mathrm{y}^{\prime}(\mathrm{t})\right|_{\mathrm{t}=0}=\left.\mathrm{y}^{\prime \prime}(\mathrm{t})\right|_{\mathrm{t}=0}=0,\left.\mathrm{y}(\mathrm{t})\right|_{\mathrm{t}=1}=\tau \mathrm{y}(\varsigma)
\end{array}\right.
$$

where $\mathscr{J}=[0,1], 2<\mathrm{p}, \mathrm{q} \leq 3,0<\tau, \varsigma<1$, and $\alpha, \chi: \mathscr{J} \times \mathscr{R} \times \mathscr{R} \rightarrow \mathscr{R}$ are continuous functions.

Motivated by the aforesaid discussion, in this manuscript, our target is to study the existence, uniqueness, and different kinds of Ulam-Hyers stability for the following nonlinear implicit FODE involving the Caputo derivative of fractional order:

$$
\left\{\begin{array}{l}
{ }^{c} \mathrm{D}^{\mathrm{P}} \mathrm{u}(\mathrm{t})-\alpha\left(\mathrm{t}, \mathrm{u}(\mathrm{t}),{ }^{c} \mathrm{D}^{\mathrm{p}} \mathrm{u}(\mathrm{t})\right)=0 ; \mathrm{t} \in \mathscr{J} \\
\left.\mathrm{u}(\mathrm{t})\right|_{\mathrm{t}=0}=-\left.\mathrm{u}(\mathrm{t})\right|_{\mathrm{t}=\mathrm{T}^{\prime}},\left.\mathrm{u}^{\prime}(\mathrm{t})\right|_{\mathrm{t}=0}=-\left.\mathrm{u}^{\prime}(\mathrm{t})\right|_{\mathrm{t}=\mathrm{T}^{\prime}},\left.\mathrm{u}^{\prime \prime}(\mathrm{t})\right|_{\mathrm{t}=0}=\left.\mathrm{u}^{\prime \prime \prime}(\mathrm{t})\right|_{\mathrm{t}=0}=0
\end{array}\right.
$$


where $3<\mathrm{p} \leq 4, \mathscr{J}=[0, \mathrm{~T}]$ with $\mathrm{T}>0$, and $\alpha: \mathscr{J} \times \mathscr{R} \times \mathscr{R} \rightarrow \mathscr{R}$ is a continuous function. Furthermore, we investigate the same aforementioned analysis for the proposed implicit coupled system involving the Caputo fractional order derivative. The proposed system is given by:

$$
\left\{\begin{array}{l}
{ }^{\mathrm{c}} \mathrm{D}^{\mathrm{P}} \mathrm{u}(\mathrm{t})-\alpha\left(\mathrm{t}, \mathrm{y}(\mathrm{t}),{ }^{c} \mathrm{D}^{\mathrm{P}} \mathrm{u}(\mathrm{t})\right)=0 ; \mathrm{t} \in \mathscr{J}, \\
{ }^{\mathrm{C}} \mathrm{D}^{\mathrm{q}} \mathrm{y}(\mathrm{t})-\chi\left(\mathrm{t}, \mathrm{u}(\mathrm{t}),{ }^{c} \mathrm{D}^{\mathrm{q}} \mathrm{y}(\mathrm{t})\right)=0 ; \mathrm{t} \in \mathscr{J}, \\
\left.\mathrm{u}(\mathrm{t})\right|_{\mathrm{t}=0}=-\left.\mathrm{u}(\mathrm{t})\right|_{\mathrm{t}=\mathrm{T}},\left.\mathrm{u}^{\prime}(\mathrm{t})\right|_{\mathrm{t}=0}=-\left.\mathrm{u}^{\prime}(\mathrm{t})\right|_{\mathrm{t}=\mathrm{T}},\left.\mathrm{u}^{\prime \prime}(\mathrm{t})\right|_{\mathrm{t}=0}=\left.\mathrm{u}^{\prime \prime \prime}(\mathrm{t})\right|_{\mathrm{t}=0}=0, \\
\left.\mathrm{y}(\mathrm{t})\right|_{\mathrm{t}=0}=-\left.\mathrm{y}(\mathrm{t})\right|_{\mathrm{t}=\mathrm{T}},\left.\mathrm{y}^{\prime}(\mathrm{t})\right|_{\mathrm{t}=0}=-\left.\mathrm{y}^{\prime}(\mathrm{t})\right|_{\mathrm{t}=\mathrm{T}},\left.\mathrm{y}^{\prime \prime}(\mathrm{t})\right|_{\mathrm{t}=0}=\left.\mathrm{y}^{\prime \prime \prime}(\mathrm{t})\right|_{\mathrm{t}=0}=0,
\end{array}\right.
$$

where $3<\mathrm{p}, \mathrm{q} \leq 4, \mathscr{J}=[0, \mathrm{~T}]$ with $\mathrm{T}>0$. The functions $\alpha, \chi: \mathscr{J} \times \mathscr{R} \times \mathscr{R} \rightarrow \mathscr{R}$ are continuous.

The structure of the manuscript is as follows: In Section 2, we present some basic materials needed to prove our main results. In Sections 3 and 4 , we set up some adequate conditions for the uniqueness, existence, and various kinds of the Ulam-Hyers stability of solutions to the proposed problems (3) and (4), respectively, by applying some standard fixed point principles mentioned in the Abstract. An example to illustrate our results is presented with each proposed problem in the concerned section. In Section 5, we present the conclusion of the manuscript.

\section{Preliminaries}

Here, we present some basic definitions and auxiliary results, which will be used throughout the manuscript. The following definitions are adopted from [2].

Definition 1. The Riemann-Liouville fractional integral of order $p>0$ for a continuous function $u: \mathscr{R}^{+} \rightarrow \mathscr{R}$ is defined as:

$$
\mathbf{I}^{p} u(t)=\frac{1}{\Gamma(p)} \int_{0}^{t}(t-s)^{p-1} u(s) d s,
$$

provided the integral exists.

Definition 2. Let $u: \mathscr{R}^{+} \rightarrow \mathscr{R}$ be an at least $n$-times continuously differentiable function, then the fractional derivative of order $p \in \mathscr{R}^{+}$in the sense of Caputo is defined as:

$$
{ }^{c} D^{p} u(t)=\frac{1}{\Gamma(n-p)} \int_{0}^{t} \frac{u^{(n)}(s)}{(t-s)^{p-n+1}} d s,
$$

where $n=[p]+1$ and $[p]$ denotes the integer part of real number $p$.

Lemma 1. The general solution of the following FODE of order $p>0$ :

$$
{ }^{c} D^{p} u(t)=\beta(t)
$$

is given by:

$$
\mathbf{I}^{p}\left[D^{p} u(t)\right]=\mathbf{I}^{p} \beta(t)+k_{0}+k_{1} t+k_{2} t^{2}+\cdots+k_{n-1} t^{n-1}, k_{i} \in \mathscr{R},
$$

where $n=[p]+1$ and $i=0,1, \ldots, n-1$.

The following theorems are adopted from [43].

Theorem 1. (Schaefer's fixed point theorem). Let $\mathscr{E}$ be a Banach space. Suppose that the operator $\mathscr{F}: \mathscr{E} \rightarrow$ $\mathscr{E}$ is a continuous compact mapping (or completely continuous). Moreover, suppose:

$$
\mathscr{B}=\{u \in \mathscr{E} \mid u=\kappa \mathscr{F}(u), 0<\kappa<1\}
$$

is a bounded set. Then, $\mathscr{F}$ has at least one fixed point in $\mathscr{E}$. 
Theorem 2. (Banach's fixed point theorem). Let $\mathscr{B}$ be a non-empty closed subset of a Banach space $\mathscr{E}$. Then, any contraction mapping $\mathscr{F}$ of $\mathscr{B}$ into itself has a unique fixed point.

\section{Existence, Uniqueness, and Stability Results for (3)}

\subsection{Existence and Uniqueness Results}

This section deals with the existence and uniqueness of solutions for the problems (3).

Lemma 2. Suppose $\beta \in \mathscr{C}(\mathscr{J}, \mathscr{R})$, then the equivalent Fredholm integral equation of:

$$
\left\{\begin{array}{l}
{ }^{c} D^{p} u(t)=\beta(t) ; p \in(3,4] ; t \in \mathscr{J}, \\
\left.u(t)\right|_{t=0}=-\left.\left.u(t)\right|_{t=T^{\prime}} u^{\prime}(t)\right|_{t=0}=-\left.\left.u^{\prime}(t)\right|_{t=T^{\prime}} u^{\prime \prime}(t)\right|_{t=0}=\left.u^{\prime \prime \prime}(t)\right|_{t=0}=0
\end{array}\right.
$$

is given by:

$$
u(t)=\int_{0}^{\top} \mathrm{G}_{p}(t, s) \beta(s) d s,
$$

where Green's function $\mathrm{G}_{p}(t, s)$ is given as:

$$
\mathrm{G}_{p}(t, s)= \begin{cases}-\frac{(\mathrm{T}-s)^{p-1}}{2 \Gamma(p)}+\frac{(\mathrm{T}-2 t)(\mathrm{T}-s)^{p-2}}{4 \Gamma(p-1)}, & 0 \leq t \leq s \leq \mathrm{T} \\ \frac{(t-s)^{p-1}-(\mathrm{T}-s)^{p-1} / 2}{\Gamma(p)}+\frac{(\mathrm{T}-2 t)(\mathrm{T}-s)^{p-2}}{4 \Gamma(p-1)}, & 0 \leq s \leq t \leq \mathrm{T} .\end{cases}
$$

Proof. Using Lemma 1, we have:

$$
\begin{aligned}
\mathrm{u}(\mathrm{t}) & =\mathrm{I}^{\mathrm{p}} \beta(\mathrm{t})-\mathrm{k}_{0}-\mathrm{k}_{1} \mathrm{t}-\mathrm{k}_{2} \mathrm{t}^{2}-\mathrm{k}_{3} \mathrm{t}^{3} \\
& =\frac{1}{\Gamma(\mathrm{p})} \int_{0}^{\mathrm{t}}(\mathrm{t}-\mathrm{s})^{\mathrm{p}-1} \beta(\mathrm{s}) \mathrm{ds}-\mathrm{k}_{0}-\mathrm{k}_{1} \mathrm{t}-\mathrm{k}_{2} \mathrm{t}^{2}-\mathrm{k}_{3} \mathrm{t}^{3} .
\end{aligned}
$$

Applying the boundary conditions $\mathrm{u}(0)=-\mathrm{u}(\mathrm{T}), \mathrm{u}^{\prime}(0)=-\mathrm{u}^{\prime}(\mathrm{T}),\left.\mathrm{u}^{\prime \prime}(\mathrm{t})\right|_{\mathrm{t}=0}=\left.\mathrm{u}^{\prime \prime \prime}(\mathrm{t})\right|_{\mathrm{t}=0}=0$, in (6), we get the following values:

$$
\begin{aligned}
& \mathrm{k}_{0}=\frac{1}{2 \Gamma(\mathrm{p})} \int_{0}^{\mathrm{T}}(\mathrm{T}-\mathrm{s})^{\mathrm{p}-1} \beta(\mathrm{s}) \mathrm{d} \mathrm{s}-\frac{\mathrm{T}}{4 \Gamma(\mathrm{p}-1)} \int_{0}^{\mathrm{T}}(\mathrm{T}-\mathrm{s})^{\mathrm{p}-2} \beta(\mathrm{s}) \mathrm{d} \mathrm{s}, \\
& \mathrm{k}_{1}=\frac{1}{2 \Gamma(\mathrm{p}-1)} \int_{0}^{\mathrm{T}}(\mathrm{T}-\mathrm{s})^{\mathrm{p}-2} \beta(\mathrm{s}) \mathrm{d} \mathrm{s}, \\
& \mathrm{k}_{2}=0 \\
& \mathrm{k}_{3}=0
\end{aligned}
$$

Therefore, (6) becomes:

$$
\begin{aligned}
\mathrm{u}(\mathrm{t}) & =\int_{0}^{\mathrm{t}} \frac{(\mathrm{t}-\mathrm{s})^{\mathrm{p}-1}}{\Gamma(\mathrm{p})} \beta(\mathrm{s}) \mathrm{ds}-\frac{1}{2} \int_{0}^{\mathrm{T}} \frac{(\mathrm{T}-\mathrm{s})^{\mathrm{p}-1}}{\Gamma(\mathrm{p})} \beta(\mathrm{s}) \mathrm{ds}+\frac{1}{4}(\mathrm{~T}-2 \mathrm{t}) \int_{0}^{\mathrm{T}} \frac{(\mathrm{T}-\mathrm{s})^{\mathrm{p}-2}}{\Gamma(\mathrm{p}-1)} \beta(\mathrm{s}) \mathrm{ds} \\
& =\int_{0}^{\top} \mathrm{G}_{\mathrm{p}}(\mathrm{t}, \mathrm{s}) \beta(\mathrm{s}) \mathrm{ds},
\end{aligned}
$$

where Green's function $G_{p}(t, s)$ is given in (5).

Therefore, in view of Lemma 2, the solution of the proposed problem (3) is equivalent to the following integral equation:

$$
\mathrm{u}(\mathrm{t})=\int_{0}^{\mathrm{T}} \mathrm{G}_{\mathrm{p}}(\mathrm{t}, \mathrm{s}) \alpha\left(\mathrm{s}, \mathrm{y}(\mathrm{s}),{ }^{c} \mathrm{D}^{\mathrm{p}} \mathrm{u}(\mathrm{s})\right) \mathrm{ds}, \mathrm{t} \in \mathscr{J}
$$


We use the following notion for convenience:

$$
\mathrm{v}(\mathrm{t})=\alpha\left(\mathrm{t}, \mathrm{u}(\mathrm{t}){ }^{\mathrm{C}} \mathrm{D}^{\mathrm{P}} \mathrm{u}(\mathrm{t})\right)=\alpha(\mathrm{t}, \mathrm{u}(\mathrm{t}), \mathrm{v}(\mathrm{t})) .
$$

Hence, (7) becomes:

$$
\mathrm{u}(\mathrm{t})=\int_{0}^{T} \mathrm{G}_{\mathrm{p}}(\mathrm{t}, \mathrm{s}) \mathrm{v}(\mathrm{s}) \mathrm{ds}, \mathrm{t} \in \mathscr{J} .
$$

Lemma 3. Green's function $\mathrm{G}_{p}(t, s)$ has the following properties:

$\left(A_{1}\right) \mathrm{G}_{p}(t, s)$ is continuous function on $\mathscr{J} \times \mathscr{J}$;

$\left(A_{2}\right) \max _{t \in \mathscr{J}} \int_{0}^{\top}\left|G_{p}(t, s)\right| d s \leq \frac{t^{p}}{\Gamma(p+1)}+\frac{T^{p}}{2 \Gamma(p+1)}+\frac{|\mathrm{T}-2 t| \mathrm{T}^{p-1}}{4 \Gamma(p)}=\frac{3 \mathrm{~T}^{p}}{2 \Gamma(p+1)}+\frac{\mathrm{T}^{p}}{4 \Gamma(p)}, t, s \in \mathscr{J}$.

Proof. $\left(A_{1}\right)$ : Green's function will always be continuous on $\mathscr{J} \times \mathscr{J}$.

$\left(A_{2}\right)$ : Due to Green's function, we have:

$\int_{0}^{T}\left|G_{p}(t, s)\right| d s=\left|\frac{1}{\Gamma(p)} \int_{0}^{t}(t-s)^{p-1} d s-\frac{1}{2 \Gamma(p)} \int_{0}^{T}(T-s)^{p-1} d s+\frac{(T-2 t)}{4 \Gamma(p-1)} \int_{0}^{T}(T-s)^{p-2} d s\right|$,

so, we get:

$$
\begin{aligned}
\max _{\mathrm{t} \in \mathscr{J}} \int_{0}^{\mathrm{T}}\left|\mathrm{G}_{\mathrm{p}}(\mathrm{t}, \mathrm{s})\right| \mathrm{d} \mathrm{s} & \leq \frac{\mathrm{t}^{\mathrm{p}}}{\Gamma(\mathrm{p}+1)}+\frac{\mathrm{T}^{\mathrm{p}}}{2 \Gamma(\mathrm{p}+1)}+\frac{|\mathrm{T}-2 \mathrm{t}| \mathrm{T}^{\mathrm{p}-1}}{4 \Gamma(\mathrm{p})} \\
& =\frac{3 T^{\mathrm{p}}}{2 \Gamma(\mathrm{p}+1)}+\frac{T^{\mathrm{p}}}{4 \Gamma(\mathrm{p})} .
\end{aligned}
$$

Suppose $\mathscr{E}=\mathscr{C}(\mathscr{J}, \mathscr{R})$ is a Banach space endowed with given norm:

$$
\|\mathrm{u}\|_{\mathscr{E}}=\max _{\mathrm{t} \in \mathscr{J}}\{|\mathrm{u}(\mathrm{t})|: \mathrm{t} \in \mathscr{J}\} .
$$

If $u$ is the solution of the problem (3), then:

$$
\begin{aligned}
\mathrm{u}(\mathrm{t})= & \frac{1}{\Gamma(\mathrm{p})} \int_{0}^{\mathrm{t}}(\mathrm{t}-\mathrm{s})^{\mathrm{p}-1} \mathrm{v}(\mathrm{s}) \mathrm{ds}-\frac{1}{2 \Gamma(\mathrm{p})} \int_{0}^{\mathrm{T}}(\mathrm{T}-\mathrm{s})^{\mathrm{p}-1} \mathrm{v}(\mathrm{s}) \mathrm{d} \mathrm{s} \\
& +\frac{(\mathrm{T}-2 \mathrm{t})}{4 \Gamma(\mathrm{p}-1)} \int_{0}^{\mathrm{T}}(\mathrm{T}-\mathrm{s})^{\mathrm{p}-2} \mathrm{v}(\mathrm{s}) \mathrm{ds}, \mathrm{t} \in \mathscr{J} .
\end{aligned}
$$

Now, to transform the problem (3) into a fixed point problem, define an operator $\mathscr{F}: \mathscr{E} \rightarrow \mathscr{E}$ as:

$$
\begin{aligned}
\mathscr{F}(\mathrm{u})(\mathrm{t})= & \frac{1}{\Gamma(\mathrm{p})} \int_{0}^{\mathrm{t}}(\mathrm{t}-\mathrm{s})^{\mathrm{p}-1} \mathrm{v}(\mathrm{s}) \mathrm{d} \mathrm{s}-\frac{1}{2 \Gamma(\mathrm{p})} \int_{0}^{\mathrm{T}}(\mathrm{T}-\mathrm{s})^{\mathrm{p}-1} \mathrm{v}(\mathrm{s}) \mathrm{d} \mathbf{s} \\
& +\frac{(\mathrm{T}-2 \mathrm{t})}{4 \Gamma(\mathrm{p}-1)} \int_{0}^{\mathrm{T}}(\mathrm{T}-\mathrm{s})^{\mathrm{p}-2} \mathrm{v}(\mathrm{s}) \mathrm{d} \mathrm{s}, \mathrm{t} \in \mathscr{J},
\end{aligned}
$$

where $\mathrm{v} \in \mathscr{E}$.

In the sequel, we need the following hypothesis:

Hypothesis 1 (H1). For every $t \in \mathscr{J}$ and $u, \bar{u}, v, \bar{v} \in \mathscr{R}$, there are constants $0<\mathscr{L}_{\alpha}<1$ and $\mathscr{K}_{\alpha}>0$, such that:

$$
|\alpha(t, u, v)-\alpha(t, \bar{u}, \bar{v})| \leq \mathscr{K}_{\alpha}|u-\bar{u}|+\mathscr{L}_{\alpha}|v-\bar{v}| .
$$


The first result is based on Theorem 1 and concerned with the existence of solutions for the problem (3).

Theorem 3. Let the hypothesis $H 1$ hold, and if $\mathscr{Q}<1$, then the problem (3) has at least one solution in $\mathscr{E}$.

Proof. Consider the operator $\mathscr{F}$ defined in (9), and suppose $\mathrm{a}(\mathrm{t})=\alpha(\mathrm{t}, 0,0)$ with $\mathrm{a}^{*}=$ $\max _{\mathrm{t} \in \mathscr{J}} \alpha(\mathrm{t}, 0,0)$ where $\mathrm{a}^{*}<\infty$. We have to show that the problem (3) has at least one solution.

Suppose the operator $\mathscr{F}$ is continuous, and consider a sequence $\left\{u_{n}\right\}$ such that $u_{n} \rightarrow u$ in $\mathscr{E}$ and $\mathrm{t} \in \mathscr{J}$, then:

$$
\begin{aligned}
\left|\mathscr{F}\left(\mathrm{u}_{\mathrm{n}}\right)(\mathrm{t})-\mathscr{F}(\mathrm{u})(\mathrm{t})\right| \leq & \frac{1}{\Gamma(\mathrm{p})} \int_{0}^{\mathrm{t}}(\mathrm{t}-\mathrm{s})^{\mathrm{p}-1}\left|\mathrm{v}_{\mathrm{n}}(\mathrm{s})-\mathrm{v}(\mathrm{s})\right| \mathrm{d} \mathrm{s}-\frac{1}{2 \Gamma(\mathrm{p})} \int_{0}^{\mathrm{T}}(\mathrm{T}-\mathrm{s})^{\mathrm{p}-1}\left|\mathrm{v}_{\mathrm{n}}(\mathrm{s})-\mathrm{v}(\mathrm{s})\right| \mathrm{ds} \\
& +\frac{(\mathrm{T}-2 \mathrm{t})}{4 \Gamma(\mathrm{p}-1)} \int_{0}^{\mathrm{T}}(\mathrm{T}-\mathrm{s})^{\mathrm{p}-2}\left|\mathrm{v}_{\mathrm{n}}(\mathrm{s})-\mathrm{v}(\mathrm{s})\right| \mathrm{d} \mathrm{s}
\end{aligned}
$$

where $\mathrm{v}_{\mathrm{n}}, \mathrm{v} \in \mathscr{E}$ and :

$$
\begin{gathered}
\mathrm{v}_{\mathrm{n}}=\alpha\left(\mathrm{t}, \mathrm{u}_{\mathrm{n}}(\mathrm{t}), \mathrm{v}_{\mathrm{n}}(\mathrm{t})\right), \\
\mathrm{v}=\alpha(\mathrm{t}, \mathrm{u}(\mathrm{t}), \mathrm{v}(\mathrm{t})) .
\end{gathered}
$$

By utilizing $H 1$, we have:

$$
\begin{aligned}
\left|\mathrm{v}_{\mathrm{n}}(\mathrm{t})-\mathrm{v}(\mathrm{t})\right| & =\mid \alpha\left(\mathrm{t}, \mathrm{u}_{\mathrm{n}}(\mathrm{t}), \mathrm{v}_{\mathrm{n}}(\mathrm{t})-\alpha(\mathrm{t}, \mathrm{u}(\mathrm{t}), \mathrm{v}(\mathrm{t})) \mid\right. \\
& \leq \mathscr{K}_{\alpha}\left|\mathrm{u}_{\mathrm{n}}(\mathrm{t})-\mathrm{u}(\mathrm{t})\right|+\mathscr{L}_{\alpha}\left|\mathrm{v}_{\mathrm{n}}(\mathrm{t})-\mathrm{v}(\mathrm{t})\right|
\end{aligned}
$$

and we obtain:

$$
\left|\mathrm{v}_{\mathrm{n}}(\mathrm{t})-\mathrm{v}(\mathrm{t})\right| \leq \frac{\mathscr{K}_{\alpha}}{1-\mathscr{L}_{\alpha}}\left|\mathrm{u}_{\mathrm{n}}(\mathrm{t})-\mathrm{u}(\mathrm{t})\right|
$$

Since we supposed that $\mathrm{u}_{\mathrm{n}} \rightarrow \mathrm{u}$, then $\mathrm{v}_{\mathrm{n}} \rightarrow \mathrm{v}$ as $\mathrm{n} \rightarrow \infty$ for each $\mathrm{t} \in \mathscr{J}$, by the Lebesgue dominated convergence theorem, (10) implies that:

$$
\left|\mathscr{F}\left(\mathrm{u}_{\mathrm{n}}\right)(\mathrm{t})-\mathscr{F}(\mathrm{u})(\mathrm{t})\right| \rightarrow 0 \text { as } \mathrm{n} \rightarrow \infty,
$$

hence:

$$
\left\|\mathscr{F}\left(\mathrm{u}_{\mathrm{n}}\right)-\mathscr{F}(\mathrm{u})\right\|_{\mathscr{E}} \rightarrow 0 \quad \text { as } \quad \mathrm{n} \rightarrow \infty
$$

Therefore, $\mathscr{F}$ is continuous.

Now, we show that $\mathscr{F}$ is bounded in $\mathscr{E}$. Therefore, for any $\xi^{*}>0$, there is $\mathcal{R}_{\mathbb{E}}>0$, such that:

$$
\mathbb{E}=\left\{\mathrm{u} \in \mathscr{E}:\|\mathrm{u}\|_{\mathscr{E}} \leq \xi^{*}\right\}
$$

then, we have:

$$
\|\mathscr{F}(\mathrm{u})\|_{\mathscr{E}} \leq \mathcal{R}_{\mathbb{E}}
$$

Taking the absolute of (9), it becomes:

$$
\begin{aligned}
|\mathscr{F}(\mathrm{u})(\mathrm{t})|= & \mid \frac{1}{\Gamma(\mathrm{p})} \int_{0}^{\mathrm{t}}(\mathrm{t}-\mathrm{s})^{\mathrm{p}-1} \mathrm{v}(\mathrm{s}) \mathrm{d} \mathrm{s}-\frac{1}{2 \Gamma(\mathrm{p})} \int_{0}^{\mathrm{T}}(\mathrm{T}-\mathrm{s})^{\mathrm{p}-1} \mathrm{v}(\mathrm{s}) \mathrm{ds} \\
& +\frac{(\mathrm{T}-2 \mathrm{t})}{4 \Gamma(\mathrm{p}-1)} \int_{0}^{\mathrm{T}}(\mathrm{T}-\mathrm{s})^{\mathrm{p}-2} \mathrm{v}(\mathrm{s}) \mathrm{ds} \mid
\end{aligned}
$$


Now, by $H 1$, we have:

$$
\begin{aligned}
|\mathrm{v}(\mathrm{t})| & =|\alpha(\mathrm{t}, \mathrm{u}(\mathrm{t}), \mathrm{v}(\mathrm{t}))| \\
& \leq|\alpha(\mathrm{t}, \mathrm{u}(\mathrm{t}), \mathrm{v}(\mathrm{t}))-\alpha(\mathrm{t}, 0,0)|+|\alpha(\mathrm{t}, 0,0)| \\
& \leq \mathscr{K}_{\alpha}|\mathrm{u}(\mathrm{t})|+\mathscr{L}_{\alpha}|\mathrm{v}(\mathrm{t})|+\mathrm{a}(\mathrm{t}) \\
& \leq \mathrm{a}^{*}+\mathscr{K}_{\alpha} \xi^{*}+\mathscr{L}_{\alpha}\|\mathrm{v}\|_{\mathscr{E}} .
\end{aligned}
$$

Therefore, we get:

$$
\|\mathrm{v}\|_{\mathscr{E}} \leq \frac{\mathrm{a}^{*}+\mathscr{K}_{\alpha} \xi^{*}}{1-\mathscr{L}_{\alpha}}=\mathscr{M}_{0}
$$

By using (12) and $\left(A_{2}\right),(11)$ becomes:

$$
|\mathscr{F}(\mathrm{u})(\mathrm{t})| \leq \mathscr{M}_{0}\left(\frac{3 \mathrm{~T}^{\mathrm{p}}}{2 \Gamma(\mathrm{p}+1)}+\frac{\mathrm{T}^{\mathrm{p}}}{4 \Gamma(\mathrm{p})}\right),
$$

which implies that:

$$
\|\mathscr{F}(\mathrm{u})\|_{\mathscr{E}} \leq \mathscr{M}_{0}\left(\frac{3 \mathrm{~T}^{2}}{2 \Gamma(\mathrm{p}+1)}+\frac{\mathrm{T}^{2}}{4 \Gamma(\mathrm{p})}\right)=\mathcal{R}_{\mathbb{E}}
$$

Hence, $\mathscr{F}(\mathbb{E})$ is uniformly bounded.

Now, we show that the operator $\mathscr{F}$ is equicontinuous in $\mathscr{E}$. Suppose $0<\mathrm{t}_{1}<\mathrm{t}_{2}<\mathrm{T}$, and let $\mathrm{u} \in \mathbb{E}$, then:

$$
\begin{aligned}
\left|\mathscr{F}(\mathrm{u})\left(\mathrm{t}_{2}\right)-\mathscr{F}(\mathrm{u})\left(\mathrm{t}_{1}\right)\right|= & \mid \frac{1}{\Gamma(\mathrm{p})} \int_{0}^{\mathrm{t}_{2}}\left(\mathrm{t}_{2}-\mathrm{s}\right)^{\mathrm{p}-1} \mathrm{v}(\mathrm{s}) \mathrm{ds}-\frac{\left(\mathrm{T}-2 \mathrm{t}_{2}\right)}{4 \Gamma(\mathrm{p}-1)} \int_{0}^{\mathrm{T}}(\mathrm{T}-\mathrm{s})^{\mathrm{p}-2} \mathrm{v}(\mathrm{s}) \mathrm{d} \mathbf{s} \\
& -\frac{1}{\Gamma(\mathrm{p})} \int_{0}^{\mathrm{t}_{1}}\left(\mathrm{t}_{1}-\mathrm{s}\right)^{\mathrm{p}-1} \mathrm{v}(\mathrm{s}) \mathrm{d} \mathrm{s}-\frac{\left(\mathrm{T}-2 \mathrm{t}_{1}\right)}{4 \Gamma(\mathrm{p}-1)} \int_{0}^{\mathrm{T}}(\mathrm{T}-\mathrm{s})^{\mathrm{p}-2} \mathrm{v}(\mathrm{s}) \mathrm{ds} \mid \\
\leq & \frac{1}{\Gamma(\mathrm{p})} \int_{0}^{\mathrm{t}_{1}}\left[\left(\mathrm{t}_{2}-\mathrm{s}\right)^{\mathrm{p}-1}-\left(\mathrm{t}_{1}-\mathrm{s}\right)^{\mathrm{p}-1}\right] \mathrm{v}(\mathrm{s}) \mathrm{d} \mathbf{s}|+| \frac{1}{\Gamma(\mathrm{p})} \int_{\mathrm{t}_{1}}^{\mathrm{t}_{2}}\left(\mathrm{t}_{2}-\mathrm{s}\right)^{\mathrm{p}-1} \mathrm{v}(\mathrm{s}) \mathrm{d} \mathbf{s} \mid \\
& +\left|-\frac{\left(\mathrm{t}_{2}-\mathrm{t}_{1}\right)}{2 \Gamma(\mathrm{p}-1)} \int_{0}^{\mathrm{T}}(\mathrm{T}-\mathrm{s})^{\mathrm{p}-2} \mathrm{v}(\mathrm{s}) \mathrm{ds}\right|
\end{aligned}
$$

by using (12), we get:

$$
\begin{aligned}
\left|\mathscr{F}(\mathrm{u})\left(\mathrm{t}_{2}\right)-\mathscr{F}(\mathrm{u})\left(\mathrm{t}_{1}\right)\right| \leq & \mathscr{M}_{0}\left(\frac{1}{\Gamma(\mathrm{p})}\left|\left(\mathrm{t}_{2}-\mathrm{s}\right)^{\mathrm{p}-1}-\left(\mathrm{t}_{1}-\mathrm{s}\right)^{\mathrm{p}-1}\right| \mathrm{d} \mathrm{s}+\frac{\mathrm{T}^{\mathrm{p}-1}}{\Gamma(\mathrm{p})} \int_{\mathrm{t}_{1}}^{\mathrm{t}_{2}} \mathrm{ds}\right. \\
& \left.+\frac{\left(\mathrm{t}_{2}-\mathrm{t}_{1}\right) \mathrm{T}^{\mathrm{p}-2}}{2 \Gamma(\mathrm{p}-1)} \int_{0}^{\mathrm{T}} \mathrm{d} \mathrm{s}\right) .
\end{aligned}
$$

The right-handed side of (13) tends to zero, when $t_{2} \rightarrow t_{1}$. Thus, $\mathscr{F}$ is equicontinuous and therefore completely continuous.

Finally, we consider a set $\mathscr{B} \subset \mathscr{E}$, which is defined as:

$$
\mathscr{B}=\{\mathrm{u} \in \mathscr{E}: \mathrm{u}=\kappa \mathscr{F}(\mathrm{u}), 0<\kappa<1\} .
$$

We need to prove that the set $\mathscr{B}$ is bounded. Suppose $\mathrm{u} \in \mathscr{B}$, such that:

$$
\mathrm{u}(\mathrm{t})=\kappa \mathscr{F}(\mathrm{u})(\mathrm{t}) \text {, where } \kappa \in(0,1) .
$$


Then, for every $\mathrm{t} \in \mathscr{J}$, we have:

$$
\begin{aligned}
|\mathrm{u}(\mathrm{t})|= & \mid \kappa\left(\frac{1}{\Gamma(\mathrm{p})} \int_{0}^{\mathrm{t}}(\mathrm{t}-\mathrm{s})^{\mathrm{p}-1} \mathrm{v}(\mathrm{s}) \mathrm{ds}-\frac{1}{2 \Gamma(\mathrm{p})} \int_{0}^{\mathrm{T}}(\mathrm{T}-\mathrm{s})^{\mathrm{p}-1} \mathrm{v}(\mathrm{s}) \mathrm{ds}\right. \\
& \left.+\frac{(\mathrm{T}-2 \mathrm{t})}{4 \Gamma(\mathrm{p}-1)} \int_{0}^{\mathrm{T}}(\mathrm{T}-\mathrm{s})^{\mathrm{p}-2} \mathrm{v}(\mathrm{s}) \mathrm{ds}\right) \mid \\
\leq & \frac{\mathrm{T}^{\mathrm{p}}}{\Gamma(\mathrm{p}+1)}|\mathrm{v}(\mathrm{s})|+\frac{\mathrm{T}^{\mathrm{p}}}{2 \Gamma(\mathrm{p}+1)}|\mathrm{v}(\mathrm{s})|+\frac{|\mathrm{T}-2 \mathrm{t}| \mathrm{T}^{\mathrm{p}-1}}{4 \Gamma(\mathrm{p})}|\mathrm{v}(\mathrm{s})| .
\end{aligned}
$$

Now, by $H 1$ for $\mathrm{t} \in \mathscr{J}$,

$$
\begin{aligned}
|\mathrm{v}(\mathrm{t})| & =|\alpha(\mathrm{t}, \mathrm{u}(\mathrm{t}), \mathrm{v}(\mathrm{t}))| \\
& \leq|\alpha(\mathrm{t}, \mathrm{u}(\mathrm{t}), \mathrm{v}(\mathrm{t}))-\alpha(\mathrm{t}, 0,0)|+|\alpha(\mathrm{t}, 0,0)| \\
& \leq \mathscr{K}_{\alpha}|\mathrm{u}(\mathrm{t})|+\mathscr{L}_{\alpha}|\mathrm{v}(\mathrm{t})|+\mathrm{a}(\mathrm{t}) .
\end{aligned}
$$

Therefore, we get:

$$
|\mathrm{v}(\mathrm{t})| \leq \frac{1}{1-\mathscr{L}_{\alpha}}\left(\mathrm{a}(\mathrm{t})+\mathscr{K}_{\alpha}|\mathrm{u}(\mathrm{t})|\right) .
$$

Plugging (15) in (14) and taking the maximum on both sides, it becomes:

$$
\begin{aligned}
\|\mathrm{u}\|_{\mathscr{E}} \leq & \frac{\mathrm{T}^{\mathrm{p}}\left[\frac{1}{1-\mathscr{L}_{\alpha}}\left(\mathrm{a}^{*}+\mathscr{K}_{\alpha}\|\mathrm{u}\|_{\mathscr{E}}\right)\right]}{\Gamma(\mathrm{p}+1)}+\frac{\mathrm{T}^{\mathrm{p}}\left[\frac{1}{1-\mathscr{L}_{\alpha}}\left(\mathrm{a}^{*}+\mathscr{K}_{\alpha}\|\mathrm{u}\|_{\mathscr{E}}\right)\right]}{2 \Gamma(\mathrm{p}+1)}+\frac{|\mathrm{T}-2 \mathrm{t}| \mathrm{T}^{\mathrm{p}-1}}{4 \Gamma(\mathrm{p})} \\
& \times\left[\frac{1}{1-\mathscr{L}_{\alpha}}\left(\mathrm{a}^{*}+\mathscr{K}_{\alpha}\|\mathrm{u}\|_{\mathscr{E}}\right)\right] .
\end{aligned}
$$

For simplicity, let:

$$
\begin{aligned}
& \mathscr{W}=\frac{\mathrm{T}^{\mathrm{p}} \mathrm{a}^{*}}{\left(1-\mathscr{L}_{\alpha}\right) \Gamma(\mathrm{p}+1)}+\frac{\mathrm{T}^{\mathrm{p}} \mathrm{a}^{*}}{2\left(1-\mathscr{L}_{\alpha}\right) \Gamma(\mathrm{p}+1)}+\frac{|\mathrm{T}-2 \mathrm{t}| \mathrm{T}^{\mathrm{p}-1} \mathrm{a}^{*}}{4\left(1-\mathscr{L}_{\alpha}\right) \Gamma(\mathrm{p})} \\
& \mathscr{Q}=\frac{\mathrm{T}^{\mathrm{p}} \mathscr{K}_{\alpha}}{\left(1-\mathscr{L}_{\alpha}\right) \Gamma(\mathrm{p}+1)}+\frac{\mathrm{T}^{\mathrm{p}} \mathscr{K}_{\alpha}}{2\left(1-\mathscr{L}_{\alpha}\right) \Gamma(\mathrm{p}+1)}+\frac{|\mathrm{T}-2 \mathrm{t}| \mathrm{T}^{\mathrm{p}-1} \mathscr{K}_{\alpha}}{4\left(1-\mathscr{L}_{\alpha}\right) \Gamma(\mathrm{p})} .
\end{aligned}
$$

Therefore, (16) becomes:

$$
\|\mathrm{u}\|_{\mathscr{E}} \leq \mathscr{Q}\|\mathrm{u}\|_{\mathscr{E}}+\mathscr{W}
$$

We obtain:

$$
\|\mathrm{u}\|_{\mathscr{E}} \leq \frac{\mathscr{W}}{1-\mathscr{Q}}
$$

Hence, $\mathscr{B}$ is bounded. Thus, by Theorem 1 , we get that the operator $\mathscr{F}$ has at least one fixed point. Therefore, the considered problem (3) has at least one solution in $\mathscr{E}$.

The following result is based on Theorem 2 and concerned with the uniqueness of solutions for the problem (3).

Theorem 4. Suppose that the hypothesis H1 holds. In addition, assume that:

$$
\frac{3 \top^{p} \mathscr{K}_{\alpha}}{2\left(1-\mathscr{L}_{\alpha}\right) \Gamma(p+1)}+\frac{\mathrm{T}^{p} \mathscr{K}_{\alpha}}{4\left(1-\mathscr{L}_{\alpha}\right) \Gamma(p)}<1 .
$$

Then, the problem (3) has a unique solution in $\mathscr{E}$. 
Proof. Suppose $\mathrm{u}, \overline{\mathrm{u}} \in \mathscr{E}$, and for $\mathrm{t} \in \mathscr{J}$, we have:

$$
\begin{aligned}
|\mathscr{F}(\mathrm{u})(\mathrm{t})-\mathscr{F}(\overline{\mathrm{u}})(\mathrm{t})| \leq & \frac{1}{\Gamma(\mathrm{p})} \int_{0}^{\mathrm{t}}(\mathrm{t}-\mathrm{s})^{\mathrm{p}-1}|\mathrm{v}(\mathrm{s})-\overline{\mathrm{v}}(\mathrm{s})| \mathrm{d} \mathrm{s}-\frac{1}{2 \Gamma(\mathrm{p})} \int_{0}^{\mathrm{T}}(\mathrm{T}-\mathrm{s})^{\mathrm{p}-1}|\mathrm{v}(\mathrm{s})-\overline{\mathrm{v}}(\mathrm{s})| \mathrm{d} \mathrm{s} \\
& +\frac{(\mathrm{T}-2 \mathrm{t})}{4 \Gamma(\mathrm{p}-1)} \int_{0}^{\mathrm{T}}(\mathrm{T}-\mathrm{s})^{\mathrm{p}-2}|\mathrm{v}(\mathrm{s})-\overline{\mathrm{v}}(\mathrm{s})| \mathrm{d} \mathrm{s}
\end{aligned}
$$

where v, $\overline{\mathrm{v}} \in \mathscr{E}$, such that:

$$
\begin{aligned}
\mathrm{v}(\mathrm{t}) & =\alpha(\mathrm{t}, \mathrm{u}(\mathrm{t}), \mathrm{v}(\mathrm{t})) \\
\overline{\mathrm{v}}(\mathrm{t}) & =\alpha(\mathrm{t}, \overline{\mathrm{u}}(\mathrm{t}), \overline{\mathrm{v}}(\mathrm{t})) .
\end{aligned}
$$

By use of $H 1$, we have:

$$
\begin{aligned}
|\mathrm{v}(\mathrm{t})-\overline{\mathrm{v}}(\mathrm{t})| & =|\alpha(\mathrm{s}, \mathrm{u}(\mathrm{s}), \mathrm{v}(\mathrm{s}))-\alpha(\mathrm{s}, \overline{\mathrm{u}}(\mathrm{s}), \overline{\mathrm{v}}(\mathrm{s}))| \\
& \leq \mathscr{K}_{\alpha}|\mathrm{u}(\mathrm{t})-\overline{\mathrm{u}}(\mathrm{t})|+\mathscr{L}_{\alpha}|\mathrm{v}(\mathrm{t})-\overline{\mathrm{v}}(\mathrm{t})| .
\end{aligned}
$$

Thus:

$$
|\mathrm{v}(\mathrm{t})-\overline{\mathrm{v}}(\mathrm{t})| \leq \frac{\mathscr{K}_{\alpha}}{1-\mathscr{L}_{\alpha}}|\mathrm{u}(\mathrm{t})-\overline{\mathrm{u}}(\mathrm{t})|
$$

Therefore, (18) becomes:

$$
\begin{aligned}
|\mathscr{F}(\mathrm{u})(\mathrm{t})-\mathscr{F}(\overline{\mathrm{u}})(\mathrm{t})| \leq & \frac{\mathscr{K}_{\alpha}}{1-\mathscr{L}_{\alpha}}\left(\frac{1}{\Gamma(\mathrm{p})} \int_{0}^{\mathrm{t}}(\mathrm{t}-\mathrm{s})^{\mathrm{p}-1}|\mathrm{u}(\mathrm{s})-\overline{\mathrm{u}}(\mathrm{s})| \mathrm{d} \mathrm{s}-\frac{1}{2 \Gamma(\mathrm{p})} \int_{0}^{\mathrm{T}}(\mathrm{T}-\mathrm{s})^{\mathrm{p}-1}|\mathrm{u}(\mathrm{s})-\overline{\mathrm{u}}(\mathrm{s})| \mathrm{d} \mathrm{s}\right. \\
& \left.+\frac{(\mathrm{T}-2 \mathrm{t})}{4 \Gamma(\mathrm{p}-1)} \int_{0}^{\mathrm{T}}(\mathrm{T}-\mathrm{s})^{\mathrm{p}-2}|\mathrm{u}(\mathrm{s})-\overline{\mathrm{u}}(\mathrm{s})| \mathrm{d} \mathrm{s}\right) .
\end{aligned}
$$

Now, taking the maximum on both sides, we get:

$$
\|\mathscr{F}(\mathrm{u})-\mathscr{F}(\overline{\mathrm{u}})\|_{\mathscr{E}} \leq\left(\frac{3 \mathrm{~T}^{\mathrm{p}} \mathscr{K}_{\alpha}}{2\left(1-\mathscr{L}_{\alpha}\right) \Gamma(\mathrm{p}+1)}+\frac{\mathrm{T}^{\mathrm{p}} \mathscr{K}_{\alpha}}{4\left(1-\mathscr{L}_{\alpha}\right) \Gamma(\mathrm{p})}\right)\|\mathrm{u}-\overline{\mathrm{u}}\|_{\mathscr{E}} .
$$

Hence, the operator $\mathscr{F}$ is a contraction. Thus, $\mathscr{F}$ has a unique fixed point, so the problem (3) has a unique solution.

\subsection{Ulam Stability Results}

In this section, we introduce Ulam-Hyers stability concepts for the problem (3). The following definitions were adopted from [50].

Let $\epsilon>0, \alpha: \mathscr{J} \times \mathscr{R} \times \mathscr{R} \rightarrow \mathscr{R}$ be a continuous function and $\vartheta: \mathscr{J} \rightarrow \mathscr{R}^{+}$nondecreasing. Consider the following inequalities.

$$
\begin{gathered}
\left|{ }^{\mathrm{c}} \mathrm{D}^{\mathrm{P}} \mathrm{u}(\mathrm{t})-\alpha\left(\mathrm{t}, \mathrm{u}(\mathrm{t}),{ }^{c} \mathrm{D}^{\mathrm{P}} \mathrm{u}(\mathrm{t})\right)\right| \leq \epsilon, \mathrm{t} \in \mathscr{J}, \\
\left|{ }^{\mathrm{c}} \mathrm{D}^{\mathrm{P}} \mathrm{u}(\mathrm{t})-\alpha\left(\mathrm{t}, \mathrm{u}(\mathrm{t}),{ }^{c} \mathrm{D}^{\mathrm{P}} \mathrm{u}(\mathrm{t})\right)\right| \leq \vartheta(\mathrm{t}), \mathrm{t} \in \mathscr{J}, \\
\left|{ }^{\mathrm{c}} \mathrm{D}^{\mathrm{P}} \mathrm{u}(\mathrm{t})-\alpha\left(\mathrm{t}, \mathrm{u}(\mathrm{t}),{ }^{c} \mathrm{D}^{\mathrm{P}} \mathrm{u}(\mathrm{t})\right)\right| \leq \vartheta(\mathrm{t}) \epsilon, \mathrm{t} \in \mathscr{J} .
\end{gathered}
$$

Definition 3. Problem (3) is called Ulam-Hyers stable if there is $C_{p} \in \mathscr{R}^{+}$such that for each $\epsilon>0$ and each solution $u \in \mathscr{E}$ of (19), there is a solution $v \in \mathscr{E}$ of (3) with:

$$
|u(t)-v(t)| \leq C_{p} \epsilon, t \in \mathscr{J} .
$$


Definition 4. Problem (3) is called generalized Ulam-Hyers stable if there is $\Psi \in \mathscr{C}\left(\mathscr{R}^{+}, \mathscr{R}^{+}\right), \Psi(0)=0$, such that for each solution $u \in \mathscr{E}$ of (19), there is a solution $v \in \mathscr{E}$ of (3) with:

$$
|u(t)-v(t)| \leq \Psi(\epsilon), t \in \mathscr{J} .
$$

Definition 5. Problem (3) is called Ulam-Hyers-Rassias stable with respect to $\vartheta$ if there is $C_{\vartheta} \in \mathscr{R}^{+}$, such that for each $\epsilon>0$ and each solution $u \in \mathscr{E}$ of (21), there is a solution $v \in \mathscr{E}$ of (3) with:

$$
|u(t)-v(t)| \leq C_{\vartheta} \vartheta(t) \epsilon, t \in \mathscr{J} .
$$

Definition 6. Problem (3) is called generalized Ulam-Hyers-Rassias stable with respect to $\vartheta$ if there is $C_{\vartheta} \in \mathscr{R}^{+}$, such that for each solution $u \in \mathscr{E}$ of (20), there is a solution $v \in \mathscr{E}$ of (3) with:

$$
|u(t)-v(t)| \leq C_{\vartheta} \vartheta(t), t \in \mathscr{J} .
$$

Remark 1. A function $u \in \mathscr{E}$ is a solution of (19) if there is $\omega_{\alpha} \in \mathscr{E}$ (dependent on $u$ ), such that:

$\left(b_{1}\right){ }^{c} D^{p} u(t)=\alpha\left(t, u(t),{ }^{c} D^{p} u(t)\right)+\omega_{\alpha}(t), t \in \mathscr{J} ;$

$\left(\mathrm{b}_{2}\right)\left|\omega_{\alpha}(t)\right| \leq \epsilon$, for all $t \in \mathscr{J}$.

Lemma 4. If $u \in \mathscr{E}$ is the solution of the inequality (19), then u will be the solution of the following inequality:

$$
|u(t)-m(t)| \leq\left(\frac{3 T^{p}}{2 \Gamma(p+1)}+\frac{T^{p}}{4 \Gamma(p)}\right) \epsilon
$$

Proof. Let $u$ be the solution of Inequality (19). Then, by $\left(b_{1}\right)$ of Remark 1 , we have:

$$
\left\{\begin{array}{l}
{ }^{c} D^{\mathrm{p}} \mathrm{u}(\mathrm{t})=\alpha\left(\mathrm{t}, \mathrm{u}(\mathrm{t}){ }^{c} \mathrm{D}^{\mathrm{p}} \mathrm{u}(\mathrm{t})\right)+\omega_{\alpha}, \mathrm{t} \in \mathscr{J}, \\
\left.\mathrm{u}(\mathrm{t})\right|_{\mathrm{t}=0}=-\left.\mathrm{u}(\mathrm{t})\right|_{\mathrm{t}=\mathrm{T}^{\prime}},\left.\mathrm{u}^{\prime}(\mathrm{t})\right|_{\mathrm{t}=0}=-\left.\mathrm{u}^{\prime}(\mathrm{t})\right|_{\mathrm{t}=\mathrm{T}^{\prime}},\left.\mathrm{u}^{\prime \prime}(\mathrm{t})\right|_{\mathrm{t}=0}=\left.\mathrm{u}^{\prime \prime \prime}(\mathrm{t})\right|_{\mathrm{t}=0}=0 .
\end{array}\right.
$$

Then, the solution of (22) is given as:

$$
\begin{aligned}
\mathrm{u}(\mathrm{t})= & \frac{1}{\Gamma(\mathrm{p})} \int_{0}^{\mathrm{t}}(\mathrm{t}-\mathrm{s})^{\mathrm{p}-1} \mathrm{v}(\mathrm{s}) \mathrm{d} \mathrm{s}-\frac{1}{2 \Gamma(\mathrm{p})} \int_{0}^{\mathrm{T}}(\mathrm{T}-\mathrm{s})^{\mathrm{p}-1} \mathrm{v}(\mathrm{s}) \mathrm{ds} \\
& +\frac{(\mathrm{T}-2 \mathrm{t})}{4 \Gamma(\mathrm{p}-1)} \int_{0}^{\mathrm{T}}(\mathrm{T}-\mathrm{s})^{\mathrm{p}-2} \mathrm{v}(\mathrm{s}) \mathrm{d} \mathrm{s}+\frac{1}{\Gamma(\mathrm{p})} \int_{0}^{\mathrm{t}}(\mathrm{t}-\mathrm{s})^{\mathrm{p}-1} \\
& \times \omega_{\alpha}(\mathrm{s}) \mathrm{d} \mathrm{s}-\frac{1}{2 \Gamma(\mathrm{p})} \int_{0}^{\mathrm{T}}(\mathrm{T}-\mathrm{s})^{\mathrm{p}-1} \omega_{\alpha}(\mathrm{s}) \mathrm{d} \mathrm{s}+\frac{(\mathrm{T}-2 \mathrm{t})}{4 \Gamma(\mathrm{p}-1)} \\
& \times \int_{0}^{\mathrm{T}}(\mathrm{T}-\mathrm{s})^{\mathrm{p}-2} \omega_{\alpha}(\mathrm{s}) \mathrm{d} \mathrm{s} .
\end{aligned}
$$

For simplicity, let $\mathrm{m}(\mathrm{t})$ be used for the terms that are free of $\omega_{\alpha}$, so we have:

$$
\begin{aligned}
\mathrm{m}(\mathrm{t})= & \frac{1}{\Gamma(\mathrm{p})} \int_{0}^{\mathrm{t}}(\mathrm{t}-\mathrm{s})^{\mathrm{p}-1} \mathrm{v}(\mathrm{s}) \mathrm{ds}-\frac{1}{2 \Gamma(\mathrm{p})} \int_{0}^{\mathrm{T}}(\mathrm{T}-\mathrm{s})^{\mathrm{p}-1} \mathrm{v}(\mathrm{s}) \mathrm{d} \mathrm{s} \\
& +\frac{(\mathrm{T}-2 \mathrm{t})}{4 \Gamma(\mathrm{p}-1)} \int_{0}^{\mathrm{T}}(\mathrm{T}-\mathrm{s})^{\mathrm{p}-2} \mathrm{v}(\mathrm{s}) \mathrm{d} \mathrm{s} .
\end{aligned}
$$

Therefore, (23) can be written as:

$$
\begin{aligned}
|\mathrm{u}(\mathrm{t})-\mathrm{m}(\mathrm{t})| \leq & \frac{1}{\Gamma(\mathrm{p})} \int_{0}^{\mathrm{t}}(\mathrm{t}-\mathrm{s})^{\mathrm{p}-1}\left|\omega_{\alpha}(\mathrm{s})\right| \mathrm{d} \mathrm{s}+\frac{1}{2 \Gamma(\mathrm{p})} \int_{0}^{\mathrm{T}}(\mathrm{T}-\mathrm{s})^{\mathrm{p}-1}\left|\omega_{\alpha}(\mathrm{s})\right| \mathrm{d} \mathbf{s} \\
& +\frac{(\mathrm{T}-2 \mathrm{t})}{4 \Gamma(\mathrm{p}-1)} \int_{0}^{\mathrm{T}}(\mathrm{T}-\mathrm{s})^{\mathrm{p}-2}\left|\omega_{\alpha}(\mathrm{s})\right| \mathrm{d} \mathbf{s} .
\end{aligned}
$$


By using $\left(b_{2}\right)$ of Remark 1 , we get:

$$
|\mathrm{u}(\mathrm{t})-\mathrm{m}(\mathrm{t})| \leq\left(\frac{3 \mathrm{~T}^{\mathrm{p}}}{2 \Gamma(\mathrm{p}+1)}+\frac{\mathrm{T}^{\mathrm{p}}}{4 \Gamma(\mathrm{p})}\right) \epsilon .
$$

Theorem 5. Suppose the hypothesis $H 1$ and:

$$
\frac{3 T^{p} \mathscr{K}_{\alpha}}{2\left(1-\mathscr{L}_{\alpha}\right) \Gamma(p+1)}+\frac{\mathrm{T}^{p} \mathscr{K}_{\alpha}}{4\left(1-\mathscr{L}_{\alpha}\right) \Gamma(p)}<1
$$

hold. Then, Problem (3) will be Ulam-Hyers stable and generalized Ulam-Hyers stable.

Proof. Suppose $u \in \mathscr{E}$ is any solution of the inequality (19) and $\mathrm{v}$ is the solution of the considered problem (3), then:

$$
\left\{\begin{array}{l}
{ }^{c} \mathrm{D}^{\mathrm{p}} \mathrm{v}(\mathrm{t})-\alpha\left(\mathrm{t}, \mathrm{v}(\mathrm{t}),{ }^{c} \mathrm{D}^{\mathrm{p}} \mathrm{v}(\mathrm{t})\right)=0 ; \mathrm{t} \in \mathscr{J}, \\
\left.\mathrm{v}(\mathrm{t})\right|_{\mathrm{t}=0}=-\left.\mathrm{v}(\mathrm{t})\right|_{\mathrm{t}=\mathrm{T}},\left.\mathrm{v}^{\prime}(\mathrm{t})\right|_{\mathrm{t}=0}=-\left.\mathrm{v}^{\prime}(\mathrm{t})\right|_{\mathrm{t}=\mathrm{T}^{\prime}},\left.\mathrm{v}^{\prime \prime}(\mathrm{t})\right|_{\mathrm{t}=0}=\left.\mathrm{v}^{\prime \prime \prime}(\mathrm{t})\right|_{\mathrm{t}=0}=0 .
\end{array}\right.
$$

Let:

$$
|\mathrm{u}(\mathrm{t})-\mathrm{v}(\mathrm{t})| \leq|\mathrm{u}(\mathrm{t})-\mathrm{m}(\mathrm{t})|+|\mathrm{m}(\mathrm{t})-\mathrm{v}(\mathrm{t})|
$$

Utilizing Lemma 4 in (25), we have:

$$
\begin{aligned}
|\mathrm{u}(\mathrm{t})-\mathrm{v}(\mathrm{t})| \leq & \left(\frac{3 T^{\mathrm{p}}}{2 \Gamma(\mathrm{p}+1)}+\frac{\mathrm{T}^{\mathrm{p}}}{4 \Gamma(\mathrm{p})}\right) \epsilon+\mid \frac{1}{\Gamma(\mathrm{p})} \int_{0}^{\mathrm{t}}(\mathrm{t}-\mathrm{s})^{\mathrm{p}-1}\left(\mathrm{y}_{\mathrm{u}}(\mathrm{s})-\mathrm{g}_{\mathrm{v}}(\mathrm{s})\right) \mathrm{d} \mathbf{s}-\frac{1}{2 \Gamma(\mathrm{p})} \int_{0}^{\mathrm{T}}(\mathrm{T}-\mathrm{s})^{\mathrm{p}-1} \\
& \times\left(\mathrm{y}_{\mathrm{u}}(\mathrm{s})-\mathrm{g}_{\mathrm{v}}(\mathrm{s})\right) \mathrm{d} \mathrm{s}+\frac{(\mathrm{T}-2 \mathrm{t})}{4 \Gamma(\mathrm{p}-1)} \int_{0}^{\mathrm{T}}(\mathrm{T}-\mathrm{s})^{\mathrm{p}-2}\left(\mathrm{y}_{\mathrm{u}}(\mathrm{s})-\mathrm{g}_{\mathrm{v}}(\mathrm{s})\right) \mathrm{ds} \mid
\end{aligned}
$$

We get:

$$
|\mathrm{u}(\mathrm{t})-\mathrm{v}(\mathrm{t})| \leq\left(\frac{3 T^{\mathrm{p}}}{2 \Gamma(\mathrm{p}+1)}+\frac{\mathrm{T}^{\mathrm{p}}}{4 \Gamma(\mathrm{p})}\right) \epsilon+\left(\frac{3 T^{\mathrm{p}}}{2 \Gamma(\mathrm{p}+1)}+\frac{\mathrm{T}^{\mathrm{p}}}{4 \Gamma(\mathrm{p})}\right)\left|\mathrm{y}_{\mathrm{u}}(\mathrm{s})-\mathrm{g}_{\mathrm{v}}(\mathrm{s})\right| .
$$

For $\mathrm{y}_{\mathrm{u}}, \mathrm{g}_{\mathrm{v}} \in \mathscr{E}$, where:

$$
\begin{aligned}
& \mathrm{y}_{\mathrm{u}}(\mathrm{t})=\alpha\left(\mathrm{t}, \mathrm{u}(\mathrm{t}), \mathrm{y}_{\mathrm{u}}(\mathrm{t})\right), \\
& \mathrm{g}_{\mathrm{v}}(\mathrm{t})=\alpha\left(\mathrm{t}, \mathrm{v}(\mathrm{t}), \mathrm{g}_{\mathrm{v}}(\mathrm{t})\right)
\end{aligned}
$$

By $H 1$ :

$$
\begin{aligned}
\left|\mathrm{y}_{\mathrm{u}}(\mathrm{t})-\mathrm{g}_{\mathrm{v}}(\mathrm{t})\right| & =\left|\alpha\left(\mathrm{t}, \mathrm{u}(\mathrm{t}), \mathrm{y}_{\mathrm{u}}(\mathrm{t})\right)+\alpha\left(\mathrm{t}, \mathrm{v}(\mathrm{t}), \mathrm{g}_{\mathrm{v}}(\mathrm{t})\right)\right| \\
& \leq \mathscr{K}_{\alpha}|\mathrm{u}(\mathrm{t})-\mathrm{v}(\mathrm{t})|+\mathscr{L}_{\alpha}\left|\mathrm{y}_{\mathrm{u}}(\mathrm{t})-\mathrm{g}_{\mathrm{v}}(\mathrm{t})\right| .
\end{aligned}
$$

We get:

$$
\left|\mathrm{y}_{\mathrm{u}}(\mathrm{t})-\mathrm{g}_{\mathrm{v}}(\mathrm{t})\right| \leq \frac{\mathscr{K}_{\alpha}}{1-\mathscr{L}_{\alpha}}|\mathrm{u}(\mathrm{t})-\mathrm{v}(\mathrm{t})| .
$$

For $\mathrm{t} \in \mathscr{J}$ and utilizing (27), (26) implies that:

$$
\|\mathrm{u}-\mathrm{v}\|_{\mathscr{E}} \leq\left(\frac{3 \mathrm{~T}^{\mathrm{p}}}{2 \Gamma(\mathrm{p}+1)}+\frac{\mathrm{T}^{\mathrm{p}}}{4 \Gamma(\mathrm{p})}\right) \epsilon+\left(\frac{3 \mathrm{~T}^{\mathrm{p}} \mathscr{K}_{\alpha}}{2\left(1-\mathscr{L}_{\alpha}\right) \Gamma(\mathrm{p}+1)}+\frac{\mathrm{T}^{\mathrm{p}} \mathscr{K}_{\alpha}}{4\left(1-\mathscr{L}_{\alpha}\right) \Gamma(\mathrm{p})}\right)\|\mathrm{u}-\mathrm{v}\|_{\mathscr{E}}
$$


After simplification, we get:

$$
\|\mathrm{u}-\mathrm{v}\|_{\mathscr{E}} \leq\left[\frac{\frac{3 \mathrm{~T}^{\mathrm{p}}}{2 \Gamma(\mathrm{p}+1)}+\frac{\mathrm{T}^{\mathrm{p}}}{4 \Gamma(\mathrm{p})}}{1-\frac{3 \mathrm{~T}_{\alpha} \mathscr{K}_{\alpha}}{2\left(1-\mathscr{L}_{\alpha}\right) \Gamma(\mathrm{p}+1)}+\frac{\mathrm{Tp}^{\mathrm{p}} \mathscr{K}_{\alpha}}{4\left(1-\mathscr{L}_{\alpha}\right) \Gamma(\mathrm{p})}}\right] \epsilon .
$$

Thus, we have:

$$
|\mathrm{u}(\mathrm{t})-\mathrm{v}(\mathrm{t})| \leq C_{\mathrm{p}} \epsilon
$$

where:

$$
C_{\mathrm{p}}=\frac{\frac{3 \mathrm{~T}^{\mathrm{p}}}{2 \Gamma \mathrm{p}+1)}+\frac{\mathrm{T}^{\mathrm{p}}}{4 \Gamma(\mathrm{p})}}{1-\frac{3 \mathrm{~T}^{\mathrm{p}} \mathscr{K}_{\alpha}}{2\left(1-\mathscr{L}_{\alpha}\right) \Gamma(\mathrm{p}+1)}+\frac{\mathrm{T}^{\mathrm{p}} \mathscr{K}_{\alpha}}{4\left(1-\mathscr{L}_{\alpha}\right) \Gamma(\mathrm{p})}} .
$$

Thus, the problem (3) is Ulam-Hyers stable.

Now, by putting $\Psi(\epsilon)=C_{\mathrm{p}} \epsilon, \Psi(0)=0$ yields that the problem (3) is generalized Ulam-Hyers stable. This completes the proof.

Hypothesis 2 (H2). Suppose an increasing function $\vartheta_{p} \in\left(\mathscr{J}, \mathscr{R}^{+}\right)$and $t \in \mathscr{J}$. Then, there is $\mu_{\vartheta, p}>0$, such that the given integral inequalities:

$$
\mathbf{I}^{p} \vartheta_{p}(t) \leq \mu_{\vartheta, p} \vartheta_{p}(t) ; \text { consequently } \mathbf{I}^{p-1} \vartheta_{p}(t) \leq \mu_{\vartheta, p} \vartheta_{p}(t)
$$

holds.

Remark 2. Under the hypotheses H1 and H2 and condition (24), Problem (3) will be Ulam-Hyers-Rassias and generalized Ulam-Hyers-Rassias stable.

\subsection{Example}

\section{Example 2.}

$$
\left\{\begin{array}{l}
{ }^{c} D^{\frac{7}{2}} u(t)=\frac{|u(t)|}{30(t+2)(1+|u(t)|)}+\frac{\left.\cos \right|^{c} D^{\frac{7}{2}} u(t) \mid}{30+t^{2}}, t \in[0,1], \\
u(0)=-u(1), u^{\prime}(0)=-u^{\prime}(1), u^{\prime \prime}(0)=u^{\prime \prime \prime}(0)=0,
\end{array}\right.
$$

where $p=\frac{7}{2}$ and $\mathrm{T}=1$. Now, for any $u, \bar{u}, v, \bar{v} \in \mathscr{R}$ and $t \in[0,1]$, we get:

$$
|\alpha(t, u, v)-\alpha(t, \bar{u}, \bar{v})| \leq \frac{1}{30}|u-\bar{u}|+\frac{1}{30}|v-\bar{v}| .
$$

Therefore, $H 1$ is satisfied with $\mathscr{K}_{\alpha}=\mathscr{L}_{\alpha}=\frac{1}{30}$.

Furthermore, plug $\mathrm{T}=1, p=\frac{7}{2}$ and $\mathscr{K}_{\alpha}=\mathscr{L}_{\alpha}=\frac{1}{30}$ in (17); we get:

$$
\left(\frac{3 T^{p} \mathscr{K}_{\alpha}}{2\left(1-\mathscr{L}_{\alpha}\right) \Gamma(p+1)}+\frac{T^{p} \mathscr{K}_{\alpha}}{4\left(1-\mathscr{L}_{\alpha}\right) \Gamma(p)}\right) \approx 0.007040789549<1 .
$$

The solution of the problem (28) is unique, and the problem is Ulam-Hyers, generalized Ulam-Hyers stable, Ulam-Hyers-Rassias stable, and generalized Ulam-Hyers-Rassias stable.

\section{Existence, Uniqueness, and Stability Results for (4)}

\subsection{Existence and Uniqueness Results}

In this section, we will investigate coupled systems of nonlinear implicit FODE. 
Lemma 5. For any $\beta, \gamma \in \mathscr{C}(\mathscr{J}, \mathscr{R})$, then the problem:

$$
\left\{\begin{array}{l}
{ }^{c} D^{p} u(t)=\beta(t) ; p \in(3,4], t \in \mathscr{J}, \\
{ }^{c} D^{q} y(t)=\gamma(t) ; q \in(3,4], t \in \mathscr{J}, \\
\left.u(t)\right|_{t=0}=-\left.u(t)\right|_{t=T^{\prime}},\left.u^{\prime}(t)\right|_{t=0}=-\left.u^{\prime}(t)\right|_{t=T^{\prime}},\left.u^{\prime \prime}(t)\right|_{t=0}=\left.u^{\prime \prime \prime}(t)\right|_{t=0}=0, \\
\left.y(t)\right|_{t=0}=-\left.\left.y(t)\right|_{t=T^{\prime}} y^{\prime}(t)\right|_{t=0}=-\left.y^{\prime}(t)\right|_{t=T^{\prime}},\left.y^{\prime \prime}(t)\right|_{t=0}=\left.y^{\prime \prime \prime}(t)\right|_{t=0}=0
\end{array}\right.
$$

has a solution if and only if:

$$
\left\{\begin{array}{l}
u(t)=\int_{0}^{\top} G_{p}(t, s) \beta(s) d s, t \in \mathscr{J}, \\
y(t)=\int_{0}^{\top} G_{q}(t, s) \gamma(s) d s, t \in \mathscr{J},
\end{array}\right.
$$

where $\mathrm{G}_{p}$ and $\mathrm{G}_{q}$ are Green's functions as given by:

$$
\begin{aligned}
& \mathrm{G}_{p}(t, s)= \begin{cases}-\frac{(\mathrm{T}-s)^{p-1}}{2 \Gamma(p)}+\frac{(\mathrm{T}-2 t)(\mathrm{T}-s)^{p-2}}{4 \Gamma(p-1)}, & 0 \leq t \leq s \leq \mathrm{T}, \\
\frac{(t-s)^{p-1}-(\mathrm{T}-s)^{p-1} / 2}{\Gamma(p)}+\frac{(\mathrm{T}-2 t)(\mathrm{T}-s)^{p-2}}{4 \Gamma(p-1)}, & 0 \leq s \leq t \leq \mathrm{T} .\end{cases} \\
& \mathrm{G}_{q}(t, s)= \begin{cases}-\frac{(\mathrm{T}-s)^{q-1}}{2 \Gamma(q)}+\frac{(\mathrm{T}-2 t)(\mathrm{T}-s)^{q-2}}{4 \Gamma(q-1)}, & 0 \leq t \leq s \leq \mathrm{T}, \\
\frac{(t-s)^{q-1}-(\mathrm{T}-s)^{q-1} / 2}{\Gamma(q)}+\frac{(\mathrm{T}-2 t)(\mathrm{T}-s)^{q-2}}{4 \Gamma(q-1)}, & 0 \leq s \leq t \leq \mathrm{T} .\end{cases}
\end{aligned}
$$

Proof. The proof is similar as given in Lemma 2.

Let $\mathscr{E}=\{\mathrm{u}(\mathrm{t}) \mid \mathrm{u} \in \mathscr{C}(\mathscr{J})\}$ be a Banach space endowed with a norm defined as $\|\mathrm{u}\|_{\mathscr{E}}=$ $\max _{\mathrm{t} \in \mathscr{J}}|\mathrm{u}(\mathrm{t})|$. Similarly, the norm defined on the product space is $\|(\mathrm{u}, \mathrm{y})\|_{\mathscr{E} \times \mathscr{E}}=\|\mathrm{u}\|_{\mathscr{E}}+\|\mathrm{y}\|_{\mathscr{E}}$. Obviously, $\left(\mathscr{E} \times \mathscr{E},\|(\mathrm{u}, \mathrm{y})\|_{\mathscr{E} \times \mathscr{E}}\right)$ is a Banach space.

Definition 7. $(u, y) \in \mathscr{E} \times \mathscr{E}$ is said to be a solution of the system (4) if $(u, y)$ satisfies the system ${ }^{c} D^{p} u(t)=$ $\alpha\left(t, y(t),{ }^{c} D^{p} u(t)\right),{ }^{c} D^{q} y(t)=\chi\left(t, u(t),{ }^{c} D^{q} y(t)\right)$ on $[0, \mathrm{~T}]$ and the conditions $u(0)=-u(\mathrm{~T}), u^{\prime}(0)=$ $-u^{\prime}(\mathrm{T}), u^{\prime \prime}(0)=u^{\prime \prime \prime}(0)=0$ and $y(0)=-y(\mathrm{~T}), y^{\prime}(0)=-y^{\prime}(\mathrm{T}), y^{\prime \prime}(0)=y^{\prime \prime \prime}(0)=0$.

Therefore, in view of Lemma 5, the solution of the proposed system (4) is equivalent to the integral equations given by:

$$
\left\{\begin{array}{l}
\mathrm{u}(\mathrm{t})=\int_{0}^{\mathrm{T}} \mathrm{G}_{\mathrm{p}}(\mathrm{t}, \mathrm{s}) \alpha\left(\mathrm{s}, \mathrm{y}(\mathrm{s}){ }^{c}{ }^{c} \mathrm{D}^{\mathrm{p}} \mathrm{u}(\mathrm{s})\right) \mathrm{ds}, \mathrm{t} \in \mathscr{J}, \\
\mathrm{y}(\mathrm{t})=\int_{0}^{\mathrm{T}} \mathrm{G}_{\mathrm{q}}(\mathrm{t}, \mathrm{s}) \chi\left(\mathrm{s}, \mathrm{u}(\mathrm{s}){ }^{c}{ }^{c} \mathrm{D}^{\mathrm{q}} \mathrm{y}(\mathrm{s})\right) \mathrm{ds}, \mathrm{t} \in \mathscr{J} .
\end{array}\right.
$$

We use the following notions for convenience:

$$
\begin{aligned}
& \mathrm{v}(\mathrm{t})=\alpha\left(\mathrm{t}, \mathrm{y}(\mathrm{t}){ }^{c} \mathrm{D}^{\mathrm{p}} \mathrm{u}(\mathrm{t})\right)=\alpha(\mathrm{t}, \mathrm{y}(\mathrm{t}), \mathrm{v}(\mathrm{t})), \\
& \mathrm{z}(\mathrm{t})=\chi\left(\mathrm{t}, \mathrm{u}(\mathrm{t}),{ }^{c} \mathrm{D}^{\mathrm{q}} \mathrm{y}(\mathrm{t})\right)=\chi(\mathrm{t}, \mathrm{u}(\mathrm{t}), \mathrm{z}(\mathrm{t})) .
\end{aligned}
$$

Hence, (30) becomes:

$$
\left\{\begin{array}{l}
\mathrm{u}(\mathrm{t})=\int_{0}^{T} \mathrm{G}_{\mathrm{p}}(\mathrm{t}, \mathrm{s}) \mathrm{v}(\mathrm{s}) \mathrm{ds}, \mathrm{t} \in \mathscr{J}, \\
\mathrm{y}(\mathrm{t})=\int_{0}^{T} \mathrm{G}_{\mathrm{q}}(\mathrm{t}, \mathrm{s}) \mathrm{z}(\mathrm{s}) \mathrm{ds}, \mathrm{t} \in \mathscr{J},
\end{array}\right.
$$

where $\mathrm{v}, \mathrm{z} \in \mathscr{E}$ satisfies the implicit functional equations. 
Lemma 6. We use $\mathrm{G}_{p, q}(t, s)=\left(\mathrm{G}_{p}(t, s), \mathrm{G}_{q}(t, s)\right)$ as the Green's function of the proposed system (4), having the following properties:

$\left(A_{3}\right) \mathrm{G}_{p, q}(t, s)$ is continuous on $\mathscr{J} \times \mathscr{J}$;

$\left(A_{4}\right) \max _{t \in \mathscr{J}} \int_{0}^{\top}\left|\mathrm{G}_{p}(t, s)\right| d s \leq \frac{t^{p}}{\Gamma(p+1)}+\frac{\mathrm{T}^{p}}{2 \Gamma(p+1)}+\frac{|\mathrm{T}-2 t| \mathrm{T}^{p-1}}{4 \Gamma(p)}=\frac{3 \mathrm{~T}^{p}}{2 \Gamma(p+1)}+\frac{\mathrm{T}^{p}}{4 \Gamma(p)}, t, s \in \mathscr{J}$, $\max _{t \in \mathscr{J}} \int_{0}^{\top}\left|G_{q}(t, s)\right| d s \leq \frac{t^{q}}{\Gamma(q+1)}+\frac{T^{q}}{2 \Gamma(q+1)}+\frac{|\mathrm{T}-2 t| \mathrm{T}^{q-1}}{4 \Gamma(q)}=\frac{3 T^{q}}{2 \Gamma(q+1)}+\frac{T^{q}}{4 \Gamma(q)}, t, s \in \mathscr{J}$.

Proof. The proofs are similar as given in Lemma 3.

For computational convenience, we introduce the following notations:

$$
\begin{aligned}
& \mathscr{S}_{\mathrm{p}}=\max \left\{\frac{3 \mathrm{~T}^{\mathrm{p}}}{2 \Gamma(\mathrm{p}+1)}+\frac{\mathrm{T}^{\mathrm{p}}}{4 \Gamma(\mathrm{p})}\right\}, \\
& \mathscr{S}_{\mathrm{q}}=\max \left\{\frac{3 \mathrm{~T}^{\mathrm{q}}}{2 \Gamma(\mathrm{q}+1)}+\frac{\mathrm{T}^{\mathrm{q}}}{4 \Gamma(\mathrm{q})}\right\}, \\
& \mathscr{L}_{\alpha}=\max \left\{\frac{3 \mathrm{~T}^{\mathrm{p}} \mathscr{K}_{\alpha}}{2\left(1-\mathscr{L}_{\alpha}\right) \Gamma(\mathrm{p}+1)}+\frac{\mathrm{T}^{\mathrm{p}} \mathscr{K}_{\alpha}}{4\left(1-\mathscr{L}_{\alpha}\right) \Gamma(\mathrm{p})}\right\}, \\
& \mathscr{Z}_{\chi}=\max \left\{\frac{3 \mathrm{~T}^{\mathrm{q}} \mathscr{K}_{\chi}}{2\left(1-\mathscr{L}_{\chi}\right) \Gamma(\mathrm{q}+1)}+\frac{\mathrm{T}^{\mathrm{q}} \mathscr{K}_{\chi}}{4\left(1-\mathscr{L}_{\chi}\right) \Gamma(\mathrm{q})}\right\} .
\end{aligned}
$$

Now, System (4) transforms into a fixed point problem. Therefore, in view of Lemma 5, an operator $\mathscr{F}: \mathscr{E} \times \mathscr{E} \rightarrow \mathscr{E} \times \mathscr{E}$ is defined as:

$$
\mathscr{F}(\mathrm{u}, \mathrm{y})(\mathrm{t})=\left(\begin{array}{l}
\int_{0}^{\mathrm{T}} \mathrm{G}_{\mathrm{p}}(\mathrm{t}, \mathrm{s}) \alpha(\mathrm{t}, \mathrm{y}(\mathrm{s}), \mathrm{v}(\mathrm{s})) \mathrm{ds} \\
\int_{0}^{\mathrm{T}} \mathrm{G}_{\mathrm{q}}(\mathrm{t}, \mathrm{s}) \chi(\mathrm{t}, \mathrm{u}(\mathrm{s}), \mathrm{z}(\mathrm{s})) \mathrm{ds}
\end{array}\right)=\left(\begin{array}{c}
\mathscr{F}_{\mathrm{p}}(\mathrm{y}, \mathrm{v})(\mathrm{t}) \\
\mathscr{F}_{\mathrm{q}}(\mathrm{u}, \mathrm{z})(\mathrm{t})
\end{array}\right)=\left(\begin{array}{c}
\mathscr{F}_{\mathrm{p}}(\mathrm{u})(\mathrm{t}) \\
\mathscr{F}_{\mathrm{q}}(\mathrm{y})(\mathrm{t})
\end{array}\right) .
$$

Then, the solution of the proposed system (4) coincides with the fixed point of the operator $\mathscr{F}$, where:

$$
\begin{aligned}
\mathscr{F}_{\mathrm{p}}(\mathrm{u})(\mathrm{t})= & \frac{1}{\Gamma(\mathrm{p})} \int_{0}^{\mathrm{t}}(\mathrm{t}-\mathrm{s})^{\mathrm{p}-1} \mathrm{v}(\mathrm{s}) \mathrm{d} \mathrm{s}-\frac{1}{2 \Gamma(\mathrm{p})} \int_{0}^{\mathrm{T}}(\mathrm{T}-\mathrm{s})^{\mathrm{p}-1} \mathrm{v}(\mathrm{s}) \mathrm{d} \mathrm{s} \\
& +\frac{(\mathrm{T}-2 \mathrm{t})}{4 \Gamma(\mathrm{p}-1)} \int_{0}^{\mathrm{T}}(\mathrm{T}-\mathrm{s})^{\mathrm{p}-2} \mathrm{v}(\mathrm{s}) \mathrm{d} \mathrm{s}, \mathrm{t} \in \mathscr{J}
\end{aligned}
$$

and:

$$
\begin{aligned}
\mathscr{F}_{\mathrm{q}}(\mathrm{y})(\mathrm{t})= & \frac{1}{\Gamma(\mathrm{q})} \int_{0}^{\mathrm{t}}(\mathrm{t}-\mathrm{s})^{\mathrm{q}-1} \mathrm{z}(\mathrm{s}) \mathrm{ds}-\frac{1}{2 \Gamma(\mathrm{q})} \int_{0}^{\mathrm{T}}(\mathrm{T}-\mathrm{s})^{\mathrm{q}-1} \mathrm{z}(\mathrm{s}) \mathrm{d} \mathrm{s} \\
& +\frac{(\mathrm{T}-2 \mathrm{t})}{4 \Gamma(\mathrm{q}-1)} \int_{0}^{\mathrm{T}}(\mathrm{T}-\mathrm{s})^{\mathrm{q}-2} \mathrm{z}(\mathrm{s}) \mathrm{d} \mathrm{s}, \mathrm{t} \in \mathscr{J} .
\end{aligned}
$$

In the sequel, we need the following hypothesis:

Hypothesis 3 (H3). For every $t \in \mathscr{J}$ and $y, v, \bar{y}, \bar{v} \in \mathscr{R}$, there are constants $\mathscr{K}_{\alpha}>0,0<\mathscr{L}_{\alpha}<1$, such that:

$$
|\alpha(t, y, v)-\alpha(t, \bar{y}, \bar{v})| \leq \mathscr{K}_{\alpha}|y-\bar{y}|+\mathscr{L}_{\alpha}|v-\bar{v}| .
$$

Similarly, for every $t \in \mathscr{J}$ and $u, z, \bar{u}, \bar{z} \in \mathscr{R}$, there are constants $\mathscr{K}_{\chi}>0,0<\mathscr{L}_{\chi}<1$, such that:

$$
|\chi(t, u, z)-\chi(t, \bar{u}, \bar{z})| \leq \mathscr{K}_{\chi}|u-\bar{u}|+\mathscr{L}_{\chi}|z-\bar{z}| .
$$


The following result is based on Theorem 1 and concerned with the existence of solutions for the problem (4).

Theorem 6. Suppose the functions $\alpha, \chi: \mathscr{J} \times \mathscr{R} \times \mathscr{R} \rightarrow \mathscr{R}$ are continuous and hypothesis $\mathrm{H} 3$ hold. In addition, it is assumed that:

$$
\mathscr{Q}_{1}<1, \quad \mathscr{Q}_{2}<1 .
$$

Then, there is at least one solution for the proposed system (4).

Proof. Since $\alpha, \chi, \mathrm{G}_{\mathrm{p}}(\mathrm{t}, \mathrm{s})$ and $\mathrm{G}_{\mathrm{q}}(\mathrm{t}, \mathrm{s})$ are continuous, so the operator $\mathscr{F}: \mathscr{E} \times \mathscr{E} \rightarrow \mathscr{E} \times \mathscr{E}$ is also continuous. Suppose $\mathrm{a}_{1}(\mathrm{t})=\alpha(\mathrm{t}, 0,0)$ with $\mathrm{a}_{1}^{*}=\max _{\mathrm{t} \in \mathscr{J}} \alpha(\mathrm{t}, 0,0)$ and $\mathrm{a}_{2}(\mathrm{t})=\chi(\mathrm{t}, 0,0)$ with $a_{2}^{*}=\max _{t \in \mathscr{J}} \chi(t, 0,0)$, where $a_{1}^{*}, a_{2}^{*}<\infty$, such that for any $\xi^{*}=\max \left\{\xi_{p}, \xi_{q}\right\}>0$, there exist $\mathcal{R}_{\mathbb{E}}>0$, such that:

$$
\mathcal{R}_{\mathbb{E}} \geq \mathscr{S}_{\mathrm{p}} \mathscr{M}^{*}+\mathscr{S}_{\mathrm{q}} \mathscr{N}^{*}
$$

We have to show that $\mathscr{F}(\mathbb{E}) \subset \mathbb{E}$, where:

$$
\mathbb{E}=\left\{(\mathrm{u}, \mathrm{y}) \in \mathscr{E} \times \mathscr{E}:\|(\mathrm{u}, \mathrm{y})\|_{\mathscr{E} \times \mathscr{E}} \leq \xi^{*}\right\},
$$

then, we have:

$$
\|\mathscr{F}(\mathrm{u}, \mathrm{y})\|_{\mathscr{E} \times \mathscr{E}} \leq \mathcal{R}_{\mathbb{E}}
$$

Then, for every $\mathrm{v} \in \mathbb{E}$, we have:

$$
\begin{aligned}
\left|\mathscr{F}_{\mathrm{p}}(\mathrm{u})(\mathrm{t})\right|= & \mid \frac{1}{\Gamma(\mathrm{p})} \int_{0}^{\mathrm{t}}(\mathrm{t}-\mathrm{s})^{\mathrm{p}-1} \mathrm{v}(\mathrm{s}) \mathrm{ds}-\frac{1}{2 \Gamma(\mathrm{p})} \int_{0}^{\mathrm{T}}(\mathrm{T}-\mathrm{s})^{\mathrm{p}-1} \mathrm{v}(\mathrm{s}) \mathrm{d} \mathbf{s} \\
& +\frac{(\mathrm{T}-2 \mathrm{t})}{4 \Gamma(\mathrm{p}-1)} \int_{0}^{\mathrm{T}}(\mathrm{T}-\mathrm{s})^{\mathrm{p}-2} \mathrm{v}(\mathrm{s}) \mathrm{d} \mathrm{s} \mid
\end{aligned}
$$

Now, by using (31) and $H 3$ in (36), we get:

$$
\left\|\mathscr{F}_{\mathrm{p}}(\mathrm{u})\right\|_{\mathscr{E}} \leq\left(\frac{3 \mathrm{~T}^{\mathrm{p}}}{2 \Gamma(\mathrm{p}+1)}+\frac{\mathrm{T}^{\mathrm{p}}}{4 \Gamma(\mathrm{p})}\right) \mathscr{M}^{*},
$$

where:

$$
\|\mathrm{v}\|_{\mathscr{E}} \leq \frac{\mathrm{a}_{1}^{*}+\mathscr{K}_{\alpha} \xi_{\mathrm{p}}}{1-\mathscr{L}_{\alpha}}=\mathscr{M}^{*}
$$

with $\|\mathrm{y}\| \leq \xi_{\mathrm{p}}$. In the same fashion, we obtain:

$$
\left\|\mathscr{F}_{\mathrm{q}}(\mathrm{y})\right\|_{\mathscr{E}} \leq\left(\frac{3 \mathrm{~T}^{\mathrm{q}}}{2 \Gamma(\mathrm{q}+1)}+\frac{\mathrm{T}^{\mathrm{q}}}{4 \Gamma(\mathrm{q})}\right) \mathscr{N}^{*},
$$

where

$$
\mathscr{N}^{*}=\frac{a_{2}^{*}+\mathscr{K}_{\chi} \xi_{\mathrm{q}}}{1-\mathscr{L}_{\chi}}
$$

with $\|\mathrm{u}\| \leq \xi_{\mathrm{q}}$. Thus, from (37) and (39), we get:

$$
\begin{aligned}
\left\|\mathscr{F}_{\mathrm{p}}(\mathrm{u})\right\|_{\mathscr{E}}+\left\|\mathscr{F}_{\mathrm{q}}(\mathrm{y})\right\|_{\mathscr{E}} & \leq\left(\frac{3 \mathrm{~T}^{\mathrm{p}}}{2 \Gamma(\mathrm{p}+1)}+\frac{\mathrm{T}^{\mathrm{p}}}{4 \Gamma(\mathrm{p})}\right) \mathscr{M}^{*}+\left(\frac{3 \mathrm{~T}^{\mathrm{q}}}{2 \Gamma(\mathrm{q}+1)}+\frac{\mathrm{T}^{\mathrm{q}}}{4 \Gamma(\mathrm{q})}\right) \mathscr{N}^{*} \\
& =\mathscr{S}_{\mathrm{p}} \mathscr{M}^{*}+\mathscr{S}_{\mathrm{q}} \mathscr{N}^{*} \leq \mathcal{R}_{\mathbb{E}}
\end{aligned}
$$

which implies that:

$$
\|\mathscr{F}(\mathrm{u}, \mathrm{y})\|_{\mathscr{E} \times \mathscr{E}} \leq \mathcal{R}_{\mathbb{E}}
$$


Thus, the operator $\mathscr{F}$ is uniformly bounded. Next, we show that the operator $\mathscr{F}$ is equicontinuous. Suppose $0<\mathrm{t}_{1}<\mathrm{t}_{2}<\mathrm{T}$ and $\mathrm{u} \in \mathbb{E}$, then:

$$
\begin{aligned}
\left|\mathscr{F}_{\mathrm{p}}(\mathrm{u})\left(\mathrm{t}_{2}\right)-\mathscr{F}_{\mathrm{p}}(\mathrm{u})\left(\mathrm{t}_{1}\right)\right|= & \mid \frac{1}{\Gamma(\mathrm{p})} \int_{0}^{\mathrm{t}_{2}}\left(\mathrm{t}_{2}-\mathrm{s}\right)^{\mathrm{p}-1} \mathrm{v}(\mathrm{s}) \mathrm{d} \mathbf{s}-\frac{\left(\mathrm{T}-2 \mathrm{t}_{2}\right)}{4 \Gamma(\mathrm{p}-1)} \int_{0}^{\mathrm{T}}(\mathrm{T}-\mathrm{s})^{\mathrm{p}-2} \mathrm{v}(\mathrm{s}) \mathrm{ds} \\
& -\frac{1}{\Gamma(\mathrm{p})} \int_{0}^{\mathrm{t}_{1}}\left(\mathrm{t}_{1}-\mathbf{s}\right)^{\mathrm{p}-1} \mathrm{v}(\mathrm{s}) \mathrm{d} \mathbf{s}-\frac{\left(\mathrm{T}-2 \mathrm{t}_{1}\right)}{4 \Gamma(\mathrm{p}-1)} \int_{0}^{\mathrm{T}}(\mathrm{T}-\mathbf{s})^{\mathrm{p}-2} \mathrm{v}(\mathbf{s}) \mathrm{ds} \mid \\
\leq & \frac{1}{\Gamma(\mathrm{p})} \int_{0}^{\mathrm{t}_{1}}\left[\left(\mathrm{t}_{2}-\mathrm{s}\right)^{\mathrm{p}-1}-\left(\mathrm{t}_{1}-\mathrm{s}\right)^{\mathrm{p}-1}\right] \mathrm{v}(\mathrm{s}) \mathrm{d} \mathrm{s}|+| \frac{1}{\Gamma(\mathrm{p})} \int_{\mathrm{t}_{1}}^{\mathrm{t}_{2}}\left(\mathrm{t}_{2}-\mathbf{s}\right)^{\mathrm{p}-1} \mathrm{v}(\mathbf{s}) \mathrm{d} \mathbf{s} \mid \\
& +\left|-\frac{\left(\mathrm{t}_{2}-\mathrm{t}_{1}\right)}{2 \Gamma(\mathrm{p}-1)} \int_{0}^{\mathrm{T}}(\mathrm{T}-\mathbf{s})^{\mathrm{p}-2} \mathrm{v}(\mathrm{s}) \mathrm{d} \mathbf{s}\right|
\end{aligned}
$$

by using (38), we get:

$$
\begin{aligned}
\left|\mathscr{F}_{\mathrm{p}}(\mathrm{u})\left(\mathrm{t}_{2}\right)-\mathscr{F}_{\mathrm{p}}(\mathrm{u})\left(\mathrm{t}_{1}\right)\right| \leq & \mathscr{M}^{*}\left(\frac{1}{\Gamma(\mathrm{p})}\left|\left(\mathrm{t}_{2}-\mathrm{s}\right)^{\mathrm{p}-1}-\left(\mathrm{t}_{1}-\mathrm{s}\right)^{\mathrm{p}-1}\right| \mathrm{d} \mathrm{s}+\frac{\mathrm{T}^{\mathrm{p}-1}}{\Gamma(\mathrm{p})} \int_{\mathrm{t}_{1}}^{\mathrm{t}_{2}} \mathrm{ds}\right. \\
& \left.+\frac{\left(\mathrm{t}_{2}-\mathrm{t}_{1}\right) \mathrm{T}^{\mathrm{p}-2}}{2 \Gamma(\mathrm{p}-1)} \int_{0}^{\mathrm{T}} \mathrm{ds}\right) .
\end{aligned}
$$

In the same fashion and by using (40), we can show that:

$$
\begin{aligned}
\left|\mathscr{F}_{\mathrm{q}}(\mathrm{y})\left(\mathrm{t}_{2}\right)-\mathscr{F}_{\mathrm{q}}(\mathrm{y})\left(\mathrm{t}_{1}\right)\right| \leq & \mathscr{N}^{*}\left(\frac{1}{\Gamma(\mathrm{q})}\left|\left(\mathrm{t}_{2}-\mathrm{s}\right)^{\mathrm{q}-1}-\left(\mathrm{t}_{1}-\mathrm{s}\right)^{\mathrm{q}-1}\right| \mathrm{ds}+\frac{\mathrm{T}^{\mathrm{q}-1}}{\Gamma(\mathrm{q})} \int_{\mathrm{t}_{1}}^{\mathrm{t}_{2}} \mathrm{ds}\right. \\
& \left.+\frac{\left(\mathrm{t}_{2}-\mathrm{t}_{1}\right) \mathrm{T}^{\mathrm{q}-2}}{2 \Gamma(\mathrm{q}-1)} \int_{0}^{\mathrm{T}} \mathrm{ds}\right)
\end{aligned}
$$

The right-hand sides of (41) and (42) tend to zero, when $t_{2} \rightarrow t_{1}$. Therefore, by the result, we infer that $\mathscr{F}$ is equicontinuous and hence uniformly equicontinuous. Therefore, the operator $\mathscr{F}$ is completely continuous.

Finally, it will be verified that the set:

$$
\mathscr{B}=\{(\mathrm{u}, \mathrm{y}) \in \mathscr{E} \times \mathscr{E} \mid(\mathrm{u}, \mathrm{y})=\kappa \mathscr{F}(\mathrm{u}, \mathrm{y}), 0<\kappa<1\}
$$

is bounded. Suppose $(\mathrm{u}, \mathrm{y}) \in \mathscr{B}$, then by definition $(\mathrm{u}, \mathrm{y})=\kappa \mathscr{F}(\mathrm{u}, \mathrm{y})$. Now, for $\mathrm{u} \in \mathscr{B}$, then in a similar way as in Theorem 3 and by using $H 3$, we have:

$$
\|\mathrm{u}\|_{\mathscr{E}} \leq \frac{\mathscr{W}_{1}}{1-\mathscr{Q}_{1}}
$$

Similarly, for $\mathrm{y} \in \mathscr{B}$, we can obtain:

$$
\|\mathrm{y}\|_{\mathscr{E}} \leq \frac{\mathscr{W}_{2}}{1-\mathscr{Q}_{2}}
$$

where:

$$
\begin{aligned}
& \mathscr{W}_{1}=\frac{\mathrm{T}^{\mathrm{p}} \mathrm{a}_{1}^{*}}{\left(1-\mathscr{L}_{\alpha}\right) \Gamma(\mathrm{p}+1)}+\frac{\mathrm{T}^{\mathrm{p}} \mathrm{a}_{1}^{*}}{2\left(1-\mathscr{L}_{\alpha}\right) \Gamma(\mathrm{p}+1)}+\frac{|\mathrm{T}-2 \mathrm{t}| \mathrm{T}^{\mathrm{p}-1} \mathrm{a}_{1}^{*}}{4\left(1-\mathscr{L}_{\alpha}\right) \Gamma(\mathrm{p})}, \\
& \mathscr{Q}_{1}=\frac{\mathrm{T}^{\mathrm{p}} \mathscr{K}_{\alpha}}{\left(1-\mathscr{L}_{\alpha}\right) \Gamma(\mathrm{p}+1)}+\frac{\mathrm{T}^{\mathrm{p}} \mathscr{K}_{\alpha}}{2\left(1-\mathscr{L}_{\alpha}\right) \Gamma(\mathrm{p}+1)}+\frac{|\mathrm{T}-2 \mathrm{t}| \mathrm{T}^{\mathrm{p}-1} \mathscr{K}_{\alpha}}{4\left(1-\mathscr{L}_{\alpha}\right) \Gamma(\mathrm{p})}, \\
& \mathscr{W}_{2}=\frac{\mathrm{T}^{\mathrm{q}} \mathrm{a}_{2}^{*}}{\left(1-\mathscr{L}_{\chi}\right) \Gamma(\mathrm{q}+1)}+\frac{\mathrm{T}^{\mathrm{q}} \mathrm{a}_{2}^{*}}{2\left(1-\mathscr{L}_{\chi}\right) \Gamma(\mathrm{q}+1)}+\frac{|\mathrm{T}-2 \mathrm{t}| \mathrm{T}^{\mathrm{q}-1} \mathrm{a}_{2}^{*}}{4\left(1-\mathscr{L}_{\chi}\right) \Gamma(\mathrm{q})}, \\
& \mathscr{Q}_{2}=\frac{\mathrm{T}^{\mathrm{q}} \mathscr{K}_{\chi}}{\left(1-\mathscr{L}_{\chi}\right) \Gamma(\mathrm{q}+1)}+\frac{\mathrm{T}^{\mathrm{q}} \mathscr{K}_{\chi}}{2\left(1-\mathscr{L}_{\chi}\right) \Gamma(\mathrm{q}+1)}+\frac{|\mathrm{T}-2 \mathrm{t}| \mathrm{T}^{\mathrm{q}-1} \mathscr{K}_{\chi}}{4\left(1-\mathscr{L}_{\chi}\right) \Gamma(\mathrm{q})} .
\end{aligned}
$$


From (43) and (44), it is implied:

$$
\|\mathrm{u}\|_{\mathscr{E}}+\|\mathrm{y}\|_{\mathscr{E}} \leq \frac{\mathscr{W}_{1}}{1-\mathscr{Q}_{1}}+\frac{\mathscr{W}_{2}}{1-\mathscr{Q}_{2}}
$$

Consequently,

$$
\|(\mathrm{u}, \mathrm{y})\|_{\mathscr{E} \times \mathscr{E}} \leq \frac{\mathscr{W}_{1}}{1-\mathscr{Q}_{1}}+\frac{\mathscr{W}_{2}}{1-\mathscr{Q}_{2}}
$$

for any $\mathrm{t} \in \mathscr{J}$, and set $\mathscr{B}$ is bounded. Hence, by Theorem 1 , the operator $\mathscr{F}$ has at least one fixed point. Thus, the proposed system (4) has at least one solution on $\mathscr{J}$.

The following result is concerned with the uniqueness of solutions for the problem (4) and is based on Theorem 2.

Theorem 7. Suppose the hypothesis H3 holds and if:

$$
\mathscr{Z}_{\alpha}+\mathscr{Z}_{\chi}<1
$$

Then, the proposed system (4) has a unique solution on $\mathscr{J}$.

Proof. Let $\mathrm{u}, \overline{\mathrm{u}} \in \mathscr{E}$, and consider:

$$
\begin{aligned}
\left|\mathscr{F}_{\mathrm{p}}(\mathrm{u})(\mathrm{t})-\mathscr{F}_{\mathrm{p}}(\overline{\mathrm{u}})(\mathrm{t})\right|= & \mid \frac{1}{\Gamma(\mathrm{p})} \int_{0}^{\mathrm{t}}(\mathrm{t}-\mathrm{s})^{\mathrm{p}-1} \mathrm{ds}-\frac{1}{2 \Gamma(\mathrm{p})} \int_{0}^{\mathrm{T}}(\mathrm{T}-\mathrm{s})^{\mathrm{p}-1} \mathrm{ds} \\
& +\frac{(\mathrm{T}-2 \mathrm{t})}{4 \Gamma(\mathrm{p}-1)} \int_{0}^{\mathrm{T}}(\mathrm{T}-\mathrm{s})^{\mathrm{p}-2} \mathrm{ds}|| \mathrm{v}(\mathrm{s})-\overline{\mathrm{v}}(\mathrm{s}) \mid .
\end{aligned}
$$

where:

$$
\begin{aligned}
\mathrm{v}(\mathrm{t}) & =\alpha(\mathrm{t}, \mathrm{y}(\mathrm{t}), \mathrm{v}(\mathrm{t})), \\
\overline{\mathrm{v}}(\mathrm{t}) & =\alpha(\mathrm{t}, \overline{\mathrm{y}}(\mathrm{t}), \overline{\mathrm{v}}(\mathrm{t})) .
\end{aligned}
$$

By using H3:

$$
\begin{aligned}
|\mathrm{v}(\mathrm{t})-\overline{\mathrm{v}}(\mathrm{t})| & =|\alpha(\mathrm{t}, \mathrm{y}(\mathrm{t}), \mathrm{v}(\mathrm{t}))-\alpha(\mathrm{t}, \overline{\mathrm{y}}(\mathrm{t}), \overline{\mathrm{v}}(\mathrm{t}))| \\
& \leq \mathscr{K}_{\alpha}|\mathrm{y}(\mathrm{t})-\overline{\mathrm{y}}(\mathrm{t})|+\mathscr{L}_{\alpha}|\mathrm{v}(\mathrm{t})-\overline{\mathrm{v}}(\mathrm{t})| .
\end{aligned}
$$

We get:

$$
|\mathrm{v}(\mathrm{t})-\overline{\mathrm{v}}(\mathrm{t})| \leq \frac{\mathscr{K}_{\alpha}}{1-\mathscr{L}_{\alpha}}|\mathrm{y}(\mathrm{t})-\overline{\mathrm{y}}(\mathrm{t})|
$$

Put (47) in (46) and taking max on both sides over $\mathscr{J}$, we get:

$$
\left\|\mathscr{F}_{\mathrm{p}}(\mathrm{u})-\mathscr{F}_{\mathrm{p}}(\overline{\mathrm{u}})\right\|_{\mathscr{E}} \leq\left(\frac{3 \mathrm{~T}^{\mathrm{p}} \mathscr{K}_{\alpha}}{2\left(1-\mathscr{L}_{\alpha}\right) \Gamma(\mathrm{p}+1)}+\frac{\mathrm{T}^{\mathrm{p}} \mathscr{K}_{\alpha}}{4\left(1-\mathscr{L}_{\alpha}\right) \Gamma(\mathrm{p})}\right)\|\mathrm{y}-\overline{\mathrm{y}}\|_{\mathscr{E}} .
$$

In the same fashion, we can obtain:

$$
\left\|\mathscr{F}_{\mathrm{q}}(\mathrm{y})-\mathscr{F}_{\mathrm{q}}(\overline{\mathrm{y}})\right\|_{\mathscr{E}} \leq\left(\frac{3 \mathrm{~T}^{\mathrm{q}} \mathscr{K}_{\chi}}{2\left(1-\mathscr{L}_{\chi}\right) \Gamma(\mathrm{q}+1)}+\frac{\mathrm{T}^{\mathrm{q}} \mathscr{K}_{\chi}}{4\left(1-\mathscr{L}_{\alpha}\right) \Gamma(\mathrm{q})}\right)\|\mathrm{u}-\overline{\mathrm{u}}\|_{\mathscr{E}}
$$

Therefore, from (48) and (49), we get:

$$
\|\mathscr{F}(\mathrm{u}, \mathrm{y})-\mathscr{F}(\overline{\mathrm{u}}, \overline{\mathrm{y}})\|_{\mathscr{E} \times \mathscr{E}} \leq\left(\mathscr{Z}_{\alpha}+\mathscr{Z}_{\chi}\right)\|(\mathrm{u}, \mathrm{y})-(\overline{\mathrm{u}}, \overline{\mathrm{y}})\|_{\mathscr{E} \times \mathscr{E}} .
$$


Thus, $\mathscr{F}$ is a contraction. Therefore, we infer by Theorem 2 that $\mathscr{F}$ has a fixed point that is the unique solution of the considered system (4).

\subsection{Ulam Stability Results}

In the current section, we study the Ulam-Hyers stability results of the proposed system (4). The following definitions were adopted from [50].

Let $\epsilon_{\mathrm{p}}, \epsilon_{\mathrm{q}}>0, \alpha, \chi: \mathscr{J} \times \mathscr{R} \times \mathscr{R} \rightarrow \mathscr{R}$ be continuous functions, and $\Omega_{\mathrm{p}}, \Omega_{\mathrm{q}}: \mathscr{J} \rightarrow \mathscr{R}^{+}$are nondecreasing. Consider the following inequalities.

$$
\begin{gathered}
\left\{\begin{array}{l}
\left|\mathrm{D}^{\mathrm{p}} \mathrm{u}(\mathrm{t})-\alpha\left(\mathrm{t}, \mathrm{y}(\mathrm{t}), \mathrm{D}^{\mathrm{p}} \mathrm{u}(\mathrm{t})\right)\right| \leq \epsilon_{\mathrm{p}}, \mathrm{t} \in \mathscr{J}, \\
\left|\mathrm{D}^{\mathrm{q}} \mathrm{y}(\mathrm{t})-\chi\left(\mathrm{t}, \mathrm{u}(\mathrm{t}), \mathrm{D}^{\mathrm{q}} \mathrm{y}(\mathrm{t})\right)\right| \leq \epsilon_{\mathrm{q}}, \mathrm{t} \in \mathscr{J},
\end{array}\right. \\
\left\{\begin{array}{l}
\left|\mathrm{D}^{\mathrm{p}} \mathrm{u}(\mathrm{t})-\alpha\left(\mathrm{t}, \mathrm{y}(\mathrm{t}), \mathrm{D}^{\mathrm{p}} \mathrm{u}(\mathrm{t})\right)\right| \leq \Omega_{\mathrm{p}}(\mathrm{t}) \epsilon_{\mathrm{p}}, \mathrm{t} \in \mathscr{J}, \\
\left|\mathrm{D}^{\mathrm{q}} \mathrm{y}(\mathrm{t})-\chi\left(\mathrm{t}, \mathrm{u}(\mathrm{t}), \mathrm{D}^{\mathrm{q}} \mathrm{y}(\mathrm{t})\right)\right| \leq \Omega_{\mathrm{q}}(\mathrm{t}) \epsilon_{\mathrm{q}}, \mathrm{t} \in \mathscr{J},
\end{array}\right. \\
\left\{\begin{array}{l}
\left|\mathrm{D}^{\mathrm{p}} \mathrm{u}(\mathrm{t})-\alpha\left(\mathrm{t}, \mathrm{y}(\mathrm{t}), \mathrm{D}^{\mathrm{p}} \mathrm{u}(\mathrm{t})\right)\right| \leq \Omega_{\mathrm{p}}(\mathrm{t}), \mathrm{t} \in \mathscr{J}, \\
\left|\mathrm{D}^{\mathrm{q}} \mathrm{y}(\mathrm{t})-\chi\left(\mathrm{t}, \mathrm{u}(\mathrm{t}), \mathrm{D}^{\mathrm{q}} \mathrm{y}(\mathrm{t})\right)\right| \leq \Omega_{\mathrm{q}}(\mathrm{t}), \mathrm{t} \in \mathscr{J},
\end{array}\right.
\end{gathered}
$$

Definition 8. System (4) is Ulam-Hyers stable if there is $C_{p, q}=\left(C_{p}, C_{q}\right)>0$ such that for some $\epsilon=$ $\left(\epsilon_{p}, \epsilon_{q}\right)>0$ and for each solution $(u, y) \in \mathscr{E} \times \mathscr{E}$ of (50), there is a solution $(\varrho, \sigma) \in \mathscr{E} \times \mathscr{E}$ of (4) with:

$$
|(u, y)(t)-(\varrho, \sigma)(t)| \leq C_{p, q} \epsilon, t \in \mathscr{J} .
$$

Definition 9. System (4) is generalized Ulam-Hyers stable, if there is $\Theta \in \mathscr{C}\left(\mathscr{R}^{+}, \mathscr{R}^{+}\right)$with $\Theta(0)=0$, such that for each solution $(u, y) \in \mathscr{E} \times \mathscr{E}$ of (50), there is a solution $(\sigma, \varrho) \in \mathscr{E} \times \mathscr{E}$ of the system (4) that satisfies:

$$
|(u, y)(t)-(\varrho, \sigma)(t)| \leq \Theta(\epsilon), t \in \mathscr{J} .
$$

Definition 10. System (4) is Ulam-Hyers-Rassias stable with respect to $\Omega_{p, q}=\left(\Omega_{p}, \Omega_{q}\right) \in \mathscr{C}^{1}(\mathscr{J}, \mathscr{R})$, if there is constant $C_{\Omega_{p}, \Omega_{q}}=\left(C_{\Omega_{p}}, C_{\Omega_{q}}\right)>0$ such that for some $\epsilon=\left(\epsilon_{p}, \epsilon_{q}\right)>0$ and for each solution $(u, y) \in \mathscr{E} \times \mathscr{E}$ of (51), there is a solution $(\varrho, \sigma) \in \mathscr{E} \times \mathscr{E}$ of the system (4) with:

$$
|(u, y)(t)-(\varrho, \sigma)(t)| \leq C_{\Omega_{p}, \Omega_{q}} \Omega_{p, q}(t) \epsilon, t \in \mathscr{J} .
$$

Definition 11. System (4) is generalized Ulam-Hyers-Rassias stable with respect to $\Omega_{p, q}=\left(\Omega_{p}, \Omega_{q}\right) \in$ $\mathscr{C}(\mathscr{J}, \mathscr{R})$, and there is constant $C_{\Omega_{p}, \Omega_{q}}=\left(C_{\Omega_{p}}, C_{\Omega_{q}}\right)>0$, such that for each solution $(u, y) \in \mathscr{E} \times \mathscr{E}$ of (52), there is a solution $(\sigma, \varrho) \in \mathscr{E} \times \mathscr{E}$ of the system (4), which satisfies:

$$
|(u, y)(t)-(\varrho, \sigma)(t)| \leq C_{\Omega_{p}, \Omega_{q}} \Omega_{p, q}(t), t \in \mathscr{J} .
$$

Remark 3. We say that $(u, y) \in \mathscr{E} \times \mathscr{E}$ is a solution of (50), if there are functions $\omega_{\alpha}, \psi_{\chi} \in \mathscr{C}(\mathscr{J}, \mathscr{R})$ that depend on $u, y$, respectively, such that:

$\left(\mathrm{b}_{3}\right)\left|\omega_{\alpha}(t)\right| \leq \epsilon_{p},\left|\psi_{\chi}(t)\right| \leq \epsilon_{q}, t \in \mathscr{J}$;

$\left(\mathrm{b}_{4}\right)$

$$
\left\{\begin{array}{l}
D^{p} u(t)=\alpha\left(t, y(t), D^{p} u(t)\right)+\omega_{\alpha}(t), t \in \mathscr{J}, \\
D^{q} y(t)=\chi\left(t, u(t), D^{q} y(t)\right)+\psi_{\chi}(t), t \in \mathscr{J} .
\end{array}\right.
$$

Lemma 7. Consider $(u, y) \in \mathscr{E} \times \mathscr{E}$ to be the solution of the inequality (50), then:

$$
\left\{\begin{array}{l}
|u(t)-m(t)| \leq \mathscr{S}_{p} \epsilon_{p}, \quad t \in \mathscr{J}, \\
|y(t)-n(t)| \leq \mathscr{S}_{q} \epsilon_{q}, \quad t \in \mathscr{J} .
\end{array}\right.
$$


Proof. Using $\left(\mathrm{b}_{4}\right)$ of Remark 3, we have:

$$
\left\{\begin{array}{l}
{ }^{c} \mathrm{D}^{\mathrm{p}} \mathrm{u}(\mathrm{t})=\alpha\left(\mathrm{t}, \mathrm{y}(\mathrm{t}),{ }^{c} \mathrm{D}^{\mathrm{p}} \mathrm{u}(\mathrm{t})\right)+\omega_{\alpha}(\mathrm{t}), \mathrm{t} \in \mathscr{J}, \\
{ }^{c} \mathrm{D}^{\mathrm{q}} \mathrm{y}(\mathrm{t})=\chi\left(\mathrm{t}, \mathrm{u}(\mathrm{t}),{ }^{c} \mathrm{D}^{\mathrm{q}} \mathrm{y}(\mathrm{t})\right)+\psi_{\chi}(\mathrm{t}), \mathrm{t} \in \mathscr{J}, \\
\left.\mathrm{u}(\mathrm{t})\right|_{\mathrm{t}=0}=-\left.\mathrm{u}(\mathrm{t})\right|_{\mathrm{t}=\mathrm{T}},\left.\mathrm{u}^{\prime}(\mathrm{t})\right|_{\mathrm{t}=0}=-\left.\mathrm{u}^{\prime}(\mathrm{t})\right|_{\mathrm{t}=\mathrm{T}},\left.\mathrm{u}^{\prime \prime}(\mathrm{t})\right|_{\mathrm{t}=0}=\left.\mathrm{u}^{\prime \prime \prime}(\mathrm{t})\right|_{\mathrm{t}=0}=0 \\
\left.\mathrm{y}(\mathrm{t})\right|_{\mathrm{t}=0}=-\left.\mathrm{y}(\mathrm{t})\right|_{\mathrm{t}=\mathrm{T}^{\prime}},\left.\mathrm{y}^{\prime}(\mathrm{t})\right|_{\mathrm{t}=0}=-\left.\mathrm{y}^{\prime}(\mathrm{t})\right|_{\mathrm{t}=\mathrm{T}^{\prime}},\left.\mathrm{y}^{\prime \prime}(\mathrm{t})\right|_{\mathrm{t}=0}=\left.\mathrm{y}^{\prime \prime \prime}(\mathrm{t})\right|_{\mathrm{t}=0}=0
\end{array}\right.
$$

Therefore, by Lemma 1, the solution of (57) will be in the given form:

$$
\left\{\begin{array}{l}
\mathrm{u}(\mathrm{t})=\int_{0}^{T} \mathrm{G}_{\mathrm{p}}(\mathrm{T}, \mathrm{s}) \alpha\left(\mathrm{s}, \mathrm{y}(\mathrm{s}),{ }^{c} \mathrm{D}^{\mathrm{p}} \mathrm{u}(\mathrm{s})\right) \mathrm{ds}+\int_{0}^{T} \mathrm{G}_{\mathrm{p}}(\mathrm{t}, \mathrm{s}) \omega_{\alpha}(\mathrm{s}) \mathrm{ds}, \mathrm{t} \in \mathscr{J}, \\
\mathrm{y}(\mathrm{t})=\int_{0}^{T} \mathrm{G}_{\mathrm{q}}(\mathrm{t}, \mathrm{s}) \chi\left(\mathrm{s}, \mathrm{u}(\mathrm{s}),{ }^{c} \mathrm{D}^{\mathrm{q}} \mathrm{y}(\mathrm{s})\right) \mathrm{d} \mathrm{s}+\int_{0}^{T} \mathrm{G}_{\mathrm{q}}(\mathrm{t}, \mathrm{s}) \psi_{\chi}(\mathrm{s}) \mathrm{ds}, \mathrm{t} \in \mathscr{J} .
\end{array}\right.
$$

From the first equation of the system (58), we have:

$$
\begin{aligned}
\mathrm{u}(\mathrm{t})= & \frac{1}{\Gamma(\mathrm{p})} \int_{0}^{\mathrm{t}}(\mathrm{t}-\mathrm{s})^{\mathrm{p}-1} \mathrm{v}(\mathrm{s}) \mathrm{d} \mathrm{s}-\frac{1}{2 \Gamma(\mathrm{p})} \int_{0}^{\mathrm{T}}(\mathrm{T}-\mathrm{s})^{\mathrm{p}-1} \mathrm{v}(\mathrm{s}) \mathrm{d} \mathrm{s} \\
& +\frac{(\mathrm{T}-2 \mathrm{t})}{4 \Gamma(\mathrm{p}-1)} \int_{0}^{\mathrm{T}}(\mathrm{T}-\mathrm{s})^{\mathrm{p}-2} \mathrm{v}(\mathrm{s}) \mathrm{d} \mathrm{s}+\frac{1}{\Gamma(\mathrm{p})} \int_{0}^{\mathrm{t}}(\mathrm{t}-\mathrm{s})^{\mathrm{p}-1} \\
& \times \omega_{\alpha}(\mathrm{s}) \mathrm{d} \mathrm{s}-\frac{1}{2 \Gamma(\mathrm{p})} \int_{0}^{\mathrm{T}}(\mathrm{T}-\mathrm{s})^{\mathrm{p}-1} \omega_{\alpha}(\mathrm{s}) \mathrm{d} \mathrm{s}+\frac{(\mathrm{T}-2 \mathrm{t})}{4 \Gamma(\mathrm{p}-1)} \\
& \times \int_{0}^{\mathrm{T}}(\mathrm{T}-\mathrm{s})^{\mathrm{p}-2} \omega_{\alpha}(\mathrm{s}) \mathrm{d} \mathrm{s} .
\end{aligned}
$$

Therefore, (59) becomes after taking the absolute:

$$
\begin{aligned}
|\mathrm{u}(\mathrm{t})-\mathrm{m}(\mathrm{t})| \leq & \mid \frac{1}{\Gamma(\mathrm{p})} \int_{0}^{\mathrm{t}}(\mathrm{t}-\mathrm{s})^{\mathrm{p}-1} \omega_{\alpha}(\mathrm{s}) \mathrm{d} \mathbf{s}-\frac{1}{2 \Gamma(\mathrm{p})} \int_{0}^{\mathrm{T}}(\mathrm{T}-\mathrm{s})^{\mathrm{p}-1} \omega_{\alpha}(\mathrm{s}) \mathrm{d} \mathbf{s} \\
& +\frac{(\mathrm{T}-2 \mathrm{t})}{4 \Gamma(\mathrm{p}-1)} \int_{0}^{\mathrm{T}}(\mathrm{T}-\mathrm{s})^{\mathrm{p}-2} \omega_{\alpha}(\mathrm{s}) \mathrm{d} \mathbf{s} \mid
\end{aligned}
$$

where:

$$
\begin{aligned}
\mathrm{m}(\mathrm{t})= & \frac{1}{\Gamma(\mathrm{p})} \int_{0}^{\mathrm{t}}(\mathrm{t}-\mathrm{s})^{\mathrm{p}-1} \mathrm{v}(\mathrm{s}) \mathrm{ds}-\frac{1}{2 \Gamma(\mathrm{p})} \int_{0}^{\mathrm{T}}(\mathrm{T}-\mathrm{s})^{\mathrm{p}-1} \mathrm{v}(\mathrm{s}) \mathrm{ds} \\
& +\frac{(\mathrm{T}-2 \mathrm{t})}{4 \Gamma(\mathrm{p}-1)} \int_{0}^{\mathrm{T}}(\mathrm{T}-\mathrm{s})^{\mathrm{p}-2} \mathrm{v}(\mathrm{s}) \mathrm{ds} .
\end{aligned}
$$

Using $\left(b_{3}\right)$ of Remark 3 and by (31), we obtain:

$$
|\mathrm{u}(\mathrm{t})-\mathrm{m}(\mathrm{t})| \leq \mathscr{S}_{\mathrm{p}} \epsilon_{\mathrm{p}}
$$

Repeating the similar procedure for the second equation of the system (58) and using (32) and $\left(b_{3}\right)$ of Remark 3, we have:

$$
|\mathrm{y}(\mathrm{t})-\mathrm{n}(\mathrm{t})| \leq \mathscr{S}_{\mathrm{q}} \epsilon_{\mathrm{q}}
$$


where:

$$
\begin{aligned}
\mathrm{n}(\mathrm{t})= & \frac{1}{\Gamma(\mathrm{q})} \int_{0}^{\mathrm{t}}(\mathrm{t}-\mathrm{s})^{\mathrm{q}-1} \mathrm{z}(\mathrm{s}) \mathrm{ds}-\frac{1}{2 \Gamma(\mathrm{q})} \int_{0}^{\mathrm{T}}(\mathrm{T}-\mathrm{s})^{\mathrm{q}-1} \mathrm{z}(\mathrm{s}) \mathrm{ds} \\
& +\frac{(\mathrm{T}-2 \mathrm{t})}{4 \Gamma(\mathrm{q}-1)} \int_{0}^{\mathrm{T}}(\mathrm{T}-\mathrm{s})^{\mathrm{q}-2} \mathrm{z}(\mathrm{s}) \mathrm{ds} .
\end{aligned}
$$

Hence, this completes the proof.

Theorem 8. Under the hypothesis $\mathrm{H} 3$ and if:

$$
\Delta=1-\mathscr{Z}_{\alpha} \mathscr{Z}_{\chi}>0
$$

holds, then the proposed system (4) is Ulam-Hyers stable.

Proof. Suppose $(\mathrm{u}, \mathrm{y}) \in \mathscr{E} \times \mathscr{E}$ is the solution of the inequality (50) and $(\varrho, \sigma) \in \mathscr{E} \times \mathscr{E}$ is the solution to the given system:

$$
\left\{\begin{array}{l}
{ }^{c} \mathrm{D}^{\mathrm{P}} \varrho(\mathrm{t})-\alpha\left(\mathrm{t}, \sigma(\mathrm{t}),{ }^{c} \mathrm{D}^{\mathrm{p}} \varrho(\mathrm{t})\right)=0 ; \mathrm{t} \in \mathscr{J}, \\
{ }^{c} \mathrm{D}^{\mathrm{q}} \sigma(\mathrm{t})-\chi\left(\mathrm{t}, \varrho(\mathrm{t}),{ }^{c} \mathrm{D}^{\mathrm{q}} \sigma(\mathrm{t})\right)=0 ; \mathrm{t} \in \mathscr{J}, \\
\left.\varrho(\mathrm{t})\right|_{\mathrm{t}=0}=-\left.\varrho(\mathrm{t})\right|_{\mathrm{t}=\mathrm{T}},\left.\varrho^{\prime}(\mathrm{t})\right|_{\mathrm{t}=0}=-\left.\varrho^{\prime}(\mathrm{t})\right|_{\mathrm{t}=\mathrm{T}},\left.\varrho^{\prime \prime}(\mathrm{t})\right|_{\mathrm{t}=0}=\left.\varrho^{\prime \prime \prime}(\mathrm{t})\right|_{\mathrm{t}=0}=0, \\
\left.\sigma(\mathrm{t})\right|_{\mathrm{t}=0}=-\left.\sigma(\mathrm{t})\right|_{\mathrm{t}=\mathrm{T}},\left.\sigma^{\prime}(\mathrm{t})\right|_{\mathrm{t}=0}=-\left.\sigma^{\prime}(\mathrm{t})\right|_{\mathrm{t}=\mathrm{T}^{\prime}},\left.\sigma^{\prime \prime}(\mathrm{t})\right|_{\mathrm{t}=0}=\left.\sigma^{\prime \prime \prime}(\mathrm{t})\right|_{\mathrm{t}=0}=0 .
\end{array}\right.
$$

Then, in view of Lemma 1, the solution of (61) is:

$$
\left\{\begin{array}{l}
\varrho(\mathrm{t})=\int_{0}^{\mathrm{T}} \mathrm{G}_{\mathrm{p}}(\mathrm{t}, \mathrm{s}) \alpha\left(\mathrm{s}, \sigma(\mathrm{s}),{ }^{c} \mathrm{D}^{\mathrm{p}} \varrho(\mathrm{s})\right) \mathrm{ds}, \mathrm{t} \in \mathscr{J}, \\
\sigma(\mathrm{t})=\int_{0}^{\mathrm{T}} \mathrm{G}_{\mathrm{q}}(\mathrm{t}, \mathrm{s}) \chi\left(\mathrm{s}, \varrho(\mathrm{s}),{ }^{c} \mathrm{D}^{\mathrm{q}} \sigma(\mathrm{s})\right) \mathrm{d} \mathrm{s}, \mathrm{t} \in \mathscr{J} .
\end{array}\right.
$$

Consider:

$$
\begin{aligned}
|\mathrm{u}(\mathrm{t})-\varrho(\mathrm{t})| \leq & |\mathrm{u}(\mathrm{t})-\mathrm{m}(\mathrm{t})|+|\mathrm{m}(\mathrm{t})-\varrho(\mathrm{t})| \\
\leq & \mathscr{S}_{\mathrm{p}} \epsilon_{\mathrm{p}}+\mid \frac{1}{\Gamma(\mathrm{p})} \int_{0}^{\mathrm{t}}(\mathrm{t}-\mathrm{s})^{\mathrm{p}-1} \mathrm{ds}-\frac{1}{2 \Gamma(\mathrm{p})} \int_{0}^{\mathrm{T}}(\mathrm{T}-\mathrm{s})^{\mathrm{p}-1} \mathrm{ds} \\
& +\frac{(\mathrm{T}-2 \mathrm{t})}{4 \Gamma(\mathrm{p}-1)} \int_{0}^{\mathrm{T}}(\mathrm{T}-\mathrm{s})^{\mathrm{p}-2} \mathrm{ds}|| \mathrm{v}(\mathrm{s})-\mathrm{v}_{\varrho}(\mathrm{s}) \mid .
\end{aligned}
$$

where $\mathrm{v}, \mathrm{v}_{\varrho} \in \mathscr{E}$, are given:

$$
\begin{aligned}
\mathrm{v}(\mathrm{t}) & =\alpha(\mathrm{t}, \mathrm{y}(\mathrm{t}), \mathrm{v}(\mathrm{t})), \\
\mathrm{v}_{\varrho}(\mathrm{t}) & =\alpha\left(\mathrm{t}, \sigma(\mathrm{t}), \mathrm{v}_{\varrho}(\mathrm{t})\right) .
\end{aligned}
$$

By using H3:

$$
\begin{aligned}
\left|\mathrm{v}(\mathrm{t})-\mathrm{v}_{\varrho}(\mathrm{t})\right| & =\left|\alpha(\mathrm{t}, \mathrm{y}(\mathrm{t}), \mathrm{v}(\mathrm{t}))-\alpha\left(\mathrm{t}, \sigma(\mathrm{t}), \mathrm{v}_{\varrho}(\mathrm{t})\right)\right| \\
& \leq \mathscr{K}_{\alpha}|\mathrm{y}(\mathrm{t})-\sigma(\mathrm{t})|+\mathscr{L}_{\alpha}\left|\mathrm{v}(\mathrm{t})-\mathrm{v}_{\varrho}(\mathrm{t})\right| .
\end{aligned}
$$

We obtain the following:

$$
\left|\mathrm{v}(\mathrm{t})-\mathrm{v}_{\varrho}(\mathrm{t})\right| \leq \frac{\mathscr{K}_{\alpha}}{1-\mathscr{L}_{\alpha}}|\mathrm{y}(\mathrm{t})-\sigma(\mathrm{t})| .
$$


Using (31) and (63) in (62), we get:

$$
\|\mathrm{u}-\varrho\|_{\mathscr{E}} \leq \mathscr{S}_{\mathrm{p}} \epsilon_{\mathrm{p}}+\left(\frac{3 \mathrm{~T}^{\mathrm{p}} \mathscr{K}_{\alpha}}{2\left(1-\mathscr{L}_{\alpha}\right) \Gamma(\mathrm{p}+1)}+\frac{\mathrm{T}^{\mathrm{p}} \mathscr{K}_{\alpha}}{4\left(1-\mathscr{L}_{\alpha}\right) \Gamma(\mathrm{p})}\right)\|\mathrm{y}-\sigma\|_{\mathscr{E}} .
$$

After using (33) in (64), we have:

$$
\|\mathrm{u}-\varrho\|_{\mathscr{E}} \leq \mathscr{S}_{\mathrm{p}} \epsilon_{\mathrm{p}}+\mathscr{Z}_{\alpha}\|\mathrm{y}-\sigma\|_{\mathscr{E}}
$$

and similarly, we use (34), we get:

$$
\|\mathrm{y}-\sigma\|_{\mathscr{E}} \leq \mathscr{S}_{\mathrm{q}} \epsilon_{\mathrm{q}}+\mathscr{Z}_{\chi}\|\mathrm{u}-\varrho\|_{\mathscr{E}},
$$

where $\mathbf{z}, \mathbf{z}_{\sigma} \in \mathscr{E}$; we have:

$$
\begin{aligned}
\mathrm{z}(\mathrm{t}) & =\chi(\mathrm{t}, \mathrm{y}(\mathrm{t}), \mathrm{z}(\mathrm{t})), \\
\mathrm{z}_{\sigma}(\mathrm{t}) & =\chi\left(\mathrm{t}, \varrho(\mathrm{t}), \mathrm{z}_{\sigma}(\mathrm{t})\right) .
\end{aligned}
$$

From (65) and (66), we write:

$$
\begin{aligned}
& \|\mathrm{u}-\varrho\|_{\mathscr{E}}-\mathscr{Z}_{\alpha}\|\mathrm{y}-\sigma\|_{\mathscr{E}} \leq \mathscr{S}_{\mathrm{p}} \epsilon_{\mathrm{p}} \\
& \|\mathrm{y}-\sigma\|_{\mathscr{E}}-\mathscr{Z}_{\chi}\|\mathrm{u}-\varrho\|_{\mathscr{E}} \leq \mathscr{S}_{\mathrm{q}} \epsilon_{\mathrm{q}} \\
& {\left[\begin{array}{cc}
1 & -\mathscr{Z}_{\alpha} \\
-\mathscr{Z}_{\chi} & 1
\end{array}\right]\left[\begin{array}{c}
\|\mathrm{u}-\varrho\|_{\mathscr{E}} \\
\|\mathrm{y}-\sigma\|_{\mathscr{E}}
\end{array}\right] \leq\left[\begin{array}{c}
\mathscr{S}_{\mathrm{p}} \epsilon_{\mathrm{p}} \\
\mathscr{S}_{\mathrm{q}} \epsilon_{\mathrm{q}}
\end{array}\right] .}
\end{aligned}
$$

Solving the above inequality, we have:

$$
\left[\begin{array}{c}
\|\mathrm{u}-\varrho\|_{\mathscr{E}} \\
\|\mathrm{y}-\sigma\|_{\mathscr{E}}
\end{array}\right] \leq\left[\begin{array}{cc}
\frac{1}{\Delta} & \frac{\mathscr{Z}_{\alpha}}{\Delta} \\
\frac{\mathscr{Z}_{X}}{\Delta} & \frac{1}{\Delta}
\end{array}\right]\left[\begin{array}{c}
\mathscr{S}_{\mathrm{p}} \epsilon_{\mathrm{p}} \\
\mathscr{S}_{\mathrm{q}} \epsilon_{\mathrm{q}}
\end{array}\right],
$$

where:

$$
\Delta=1-\mathscr{Z}_{\alpha} \mathscr{Z}_{\chi}>0
$$

Further simplification gives:

$$
\begin{aligned}
\|\mathrm{u}-\varrho\|_{\mathscr{E}} & \leq \frac{\mathscr{S}_{\mathrm{p}} \epsilon_{\mathrm{p}}}{\Delta}+\frac{\mathscr{Z}_{\alpha} \mathscr{S}_{\mathrm{q}} \epsilon_{\mathrm{q}}}{\Delta} \\
\|\mathrm{y}-\sigma\|_{\mathscr{E}} & \leq \frac{\mathscr{S}_{\mathrm{q}} \epsilon_{\mathrm{q}}}{\Delta}+\frac{\mathscr{Z}_{\chi} \mathscr{S}_{\mathrm{p}} \epsilon_{\mathrm{p}}}{\Delta}
\end{aligned}
$$

from which we have:

$$
\|\mathrm{u}-\varrho\|_{\mathscr{E}}+\|\mathrm{y}-\sigma\|_{\mathscr{E}} \leq \frac{\mathscr{S}_{\mathrm{p}} \epsilon_{\mathrm{p}}}{\Delta}+\frac{\mathscr{S}_{\mathrm{q}} \epsilon_{\mathrm{q}}}{\Delta}+\frac{\mathscr{Z}_{\alpha} \mathscr{S}_{\mathrm{q}} \epsilon_{\mathrm{q}}}{\Delta}+\frac{\mathscr{Z}_{\chi} \mathscr{S}_{\mathrm{p}} \epsilon_{\mathrm{p}}}{\Delta} .
$$

Let $\max \left\{\epsilon_{\mathrm{p}}, \epsilon_{\mathrm{q}}\right\}=\epsilon$, then from (67), we have:

$$
\|(\mathrm{u}, \mathrm{y})-(\varrho, \sigma)\|_{\mathscr{E} \times \mathscr{E}} \leq C_{\mathrm{p}, \mathrm{q}} \epsilon,
$$


where:

$$
C_{\mathrm{p}, \mathrm{q}}=\left[\frac{\mathscr{S}_{\mathrm{p}}}{\Delta}+\frac{\mathscr{S}_{\mathrm{q}}}{\Delta}+\frac{\mathscr{Z}_{\alpha} \mathscr{S}_{\mathrm{q}}}{\Delta}+\frac{\mathscr{Z}_{\chi} \mathscr{S}_{\mathrm{p}}}{\Delta}\right] .
$$

Remark 4. By setting $\Theta(\epsilon)=C_{p, q} \epsilon, \Theta(0)=0$ in (68), then by Definition 9, the proposed system (4) is generalized Ulam-Hyers stable.

Hypothesis 4 (H4). Suppose $\Omega_{p}, \Omega_{q} \in\left(\mathscr{J}, \mathscr{R}^{+}\right)$are increasing functions. Then, there are $\Lambda_{\Omega_{p}}, \Lambda_{\Omega_{q}}>0$, such that for each $t \in \mathscr{J}$, the given integral inequalities:

$$
\mathbf{I}^{p} \Omega_{p}(t) \leq \Lambda_{\Omega_{p}} \Omega_{p}(t) ; \text { consequently } \mathbf{I}^{p-1} \Omega_{p}(t) \leq \Lambda_{\Omega_{p}} \Omega_{p}(t)
$$

and:

$$
\mathbf{I}^{q} \Omega_{q}(t) \leq \Lambda_{\Omega_{q}} \Omega_{q}(t) ; \text { consequently } \mathbf{I}^{q-1} \Omega_{q}(t) \leq \Lambda_{\Omega_{q}} \Omega_{q}(t)
$$

hold.

Remark 5. Under the hypotheses H4 and (60) and by utilizing Definitions 10 and 11, then by the same process as in Lemma 7 and Theorem 8, System (4) will be Ulam-Hyers-Rassias and generalized Ulam-Hyers-Rassias stable.

\subsection{Example}

Example 3.

$$
\left\{\begin{array}{l}
{ }^{c} D^{\frac{7}{2}} u(t)=\frac{|y(t)|}{30(t+2)(1+|y(t)|)}+\frac{\cos \left|{ }^{c} D^{\frac{7}{2}} u(t)\right|}{30+t^{2}}, t \in[0,1], \\
{ }^{c} D^{\frac{7}{2}} y(t)-\frac{2+|u(t)|+\left|{ }^{c} D^{\frac{7}{2}} y(t)\right|}{70 e^{t+2}\left(1+|u(t)|+\left.\right|^{c} D^{\frac{7}{2}} y(t) \mid\right)}=0, t \in[0,1], \\
u(0)=-u(1), u^{\prime}(0)=-u^{\prime}(1), u^{\prime \prime}(0)=u^{\prime \prime \prime}(0)=0, \\
y(0)=-y(1), y^{\prime}(0)=-y^{\prime}(1), y^{\prime \prime}(0)=y^{\prime \prime \prime}(0)=0 .
\end{array}\right.
$$

From System (69), we can see $p=q=\frac{7}{2}$ and $\mathrm{T}=1$. Furthermore, we can easily find $\mathscr{K}_{\alpha}=\mathscr{L}_{\alpha}=\frac{1}{30}$ and $\mathscr{K}_{\chi}=\mathscr{L}_{\chi}=\frac{1}{70 e^{2}}$. Therefore:

$$
\mathscr{Z}_{\alpha}+\mathscr{Z}_{\chi} \approx 0.007436314061<1
$$

Hence, the system (69) has a unique solution. Moreover,

$$
\Delta=1-\mathscr{Z}_{\alpha} \mathscr{Z}_{\chi} \approx 0.9999972152>0 .
$$

Condition (60) is also satisfied. Thus, System (69) is Ulam-Hyers, generalized Ulam-Hyers, Ulam-Hyers-Rassias, and generalized Ulam-Hyers-Rassias stable.

\section{Conclusions}

We have successfully derived some essential conditions for the existence, uniqueness, and different kinds of Ulam-Hyers stability for the solutions of the considered problems (3) and (4). The required outcomes have been obtained by utilizing standard fixed point theorems mentioned in the Abstract. Additionally, we have set up some suitable conditions for different kinds of Ulam-Hyers stability of the solutions of the aforesaid problems. For the justification, we have presented an example for each problem that supported the main results.

Author Contributions: All authors equally contributed to this manuscript. 
Funding: This research work has been supported by Theoretical and Computational Science (TaCS) Center under Computational and Applied Science for Smart Innovation research Cluster (CLASSIC), Faculty of Science, KMUTT.

Acknowledgments: We are very thankful to the anonymous referees for their careful reading and suggestions, which improved the quality of this paper.

Conflicts of Interest: There is no conflict of interests regarding this research work.

\section{References}

1. Hilfer, R. Applications of Fractional Calculus in Physics; World Scientific: Singapore, 2000, ISBN 978-981-02-3457-7.

2. Kilbas, A.A.; Srivastava, H.M.; Trujillo, J.J. Theory and Applications of Fractional Differential Equations, North-Holland Mathematics Studies; Elsevier: Amsterdam, The Netherlands, 2006; Volume 204.

3. Liu, F.; Burrage, K. Novel techniques in parameter estimation for fractional dynamical models arising from biological systems. Comput. Math. Appl. 2011, 62, 822-833. [CrossRef]

4. Meral, F.; Royston, T.; Magin, R. Fractional calculus in viscoelasticity: An experimental study. Commun. Nonlinear Sci. Numer. Simul. 2010, 15, 939-945. [CrossRef]

5. Nigmatullin, R.; Omay, T.; Baleanu, D. On fractional filtering versus conventional filtering in economics. Commun. Nonlinear Sci. Numer. Simul. 2010, 15, 979-986. [CrossRef]

6. Oldham, K. Fractional differential equations in electrochemistry. Adv. Eng. Softw. 2010, 41, 9-12. [CrossRef]

7. Podlubny, I. Fractional Differential Equations, Mathematics in Science and Engineering; Academic Press: New York, NY, USA, 1999.

8. Ahmad, B.; Nieto, J.J. Existence of solutions for nonlocal boundary value problems of higher-order nonlinear fractional differential equations. Abstr. Appl. Anal. 2009, 2009, 1-9. [CrossRef]

9. Benchohra, M.; Hamani, S.; Ntouyas, S.K. Boundary value problems for differential equations with fractional order and nonlocal conditions. Nonlinear Anal. 2009, 71, 2391-2396. [CrossRef]

10. Yang, C. Positive solutions for a three-point boundary value problem of fractional Q-Difference equations. Symmetry 2018, 10, 358. [CrossRef]

11. Agarwal, R.P.; Cabada, A.; Otero-Espinar, V.; Dontha, S. Existence and uniqueness of solutions for anti-periodic difference equations. Arch. Inequal. Appl. 2004, 2, 397-411.

12. Ahmad, B.; Nieto, J.J. Existence and approximation of solutions for a class of nonlinear impulsive functional differential equations with anti-periodic boundary conditions. Nonlinear Anal. 2008, 69, 3291-3298. [CrossRef]

13. Chen, H.L. Antiperiodic wavelets. J. Comput. Math. 1996, 14, 32-39.

14. Chen, Y.; An, H. Numerical solutions of coupled Burgers equations with time and space fractional derivatives. Appl. Math. Comput. 2008, 200, 87-95. [CrossRef]

15. Duan, J.; Chen, L. Solution of fractional differential equation systems and computation of matrix mittag-leffler functions. Symmetry 2018, 10, 503. [CrossRef]

16. Gafiychuk, V.; Datsko, B.; Meleshko, V.; Blackmore, D. Analysis of the solutions of coupled nonlinear fractional reaction-difusion equations. Chaos Soliton Fractals 2009, 41, 1095-1104. [CrossRef]

17. Lazarević, M.P. Finite time stability analysis of $\mathrm{PD}^{\alpha}$ fractional control of robotic time-delay systems. Mech. Res. Commun. 2006, 33, 269-279. [CrossRef]

18. Shao, J. Anti-periodic solutions for shunting inhibitory cellular neural networks with timevarying delays. Phys. Lett. A 2008, 372, 5011-5016. [CrossRef]

19. Wang, G.; Ahmad, B.; Zhang, L. Impulsive anti-periodic boundary value problem for nonlinear differential equations of fractional order. Nonlinear Anal. 2011, 74, 792-804. [CrossRef]

20. Ahmad, B.; Nieto, J.J. Existence of solutions for anti-periodic boundary value problems involving fractional differential equations via Leray-Schauder degree theory. Topol. Methods Nonlinear Anal. J. Juliusz Schauder Cent. 2010 35, 295-304.

21. Benavides, T.D. An existence theorem for implicit differential equations in a Banach space. Ann. Mat. Pura Appl. 1978, 118, 119-130. [CrossRef]

22. Emmanuele, G.; Ricceri, B. On the existence of solutions of ordinary differential equations in implicit form in Banach spaces. Ann. Mat. Pura Appl. 1981, 129, 367-382. (In Italian) [CrossRef] 
23. Hokkanen, V.M. Existence of a periodic solution for implicit nonlinear equations. Differ. Integral Equ. 1996, 9 , 745-760.

24. Li, D. Peano's theorem for implicit differential equations. J. Math. Anal. Appl. 2001, 258, 591-616. [CrossRef]

25. Liu, B. An anti-periodic LaSalle oscillation theorem for a class of functional differential equations. J. Comput. Appl. Math. 2009, 223, 1081-1086. [CrossRef]

26. Benchohra, M.; Lazreg, J.E. Existence and uniqueness results for nonlinear implicit fractional differential equations with boundary conditions. Rom. J. Math. Comput. Sci. 2014, 4, 60-72.

27. Benchohra, M.; Lazreg, J.E. Nonlinear fractional implicit differential equations. Commun. Appl. Anal. 2013, 17, 471-482.

28. Nieto, J.J.; Ouahab, A.; Venktesh, V. Implicit fractional differential equations via the Liouville-Caputo derivative. Mathematics. 2015, 3, 398-411. [CrossRef]

29. Ulam, S.M. A Collection of the Mathematical Problems; Interscience: New York, NY, USA, 1960.

30. Hyers, D.H. On the stability of the linear functional equation. Proc. Natl. Acad. Sci. USA 1941, 27, $222-224$. [CrossRef]

31. Hyers, D.H.; Isac, G.; Rassias, T.M. Stability of Functional Equations in Several Variables; Birkhäiuser: Boston, MA, USA, 1998.

32. Jung, S.M. Hyers-Ulam stability of linear differential equations of first order. Appl. Math. Lett. 2006, 19, 854-858. [CrossRef]

33. Zada, A.; Faisal, S.; Li, Y. On the Hyers-Ulam stability of first order impulsive delay differential equations. J. Funct. Spaces 2016, 2016, 1-6. [CrossRef]

34. Rassias, T.M. On the stability of functional equations and a problem of Ulam. Acta. Appl. Math. 2000, 62, 23-130. [CrossRef]

35. Brillouët-Belluot, N.; Brzdęk, J.; Ciepliński, K. On some recent developments in Ulam's type stability. Abstr. Appl. Anal. 2012, 2018, 716936. [CrossRef]

36. Li, M.; Wang, J.; O'Regan, D. Existence and Ulam's stability for conformable fractional differential equations with constant coefficients. Bull. Malays. Math. Sci. Soc. 2017, 2017, 1-22. [CrossRef]

37. Ahmad, N.; Ali, Z.; Shah, K.; Zada, A.; Rahman, G. Analysis of implicit type nonlinear dynamical problem of impulsive fractional differential equations. Complexity 2018, 2018, 1-15. [CrossRef]

38. Ali, Z.; Zada, A.; Shah, K. Existence and stability analysis of three point boundary value problem. Int. J. Appl. Comput. Math. 2017, 3, 651-664. [CrossRef]

39. Benchohra, M.; Bouriah, S. Existence and stability results for nonlinear boundary value problem for implicit differential equations of fractional order. Moroccan J. Pure Appl. Anal. 2015, 1, 22-37. [CrossRef]

40. Benchohra, M.; Bouriah, S.; Henderson, J. Existence and stability results for nonlinear implicit neutral fractional differential equations with finite delay and impulses. Commun. Appl. Nonlinear Anal. 2015, 22, $46-67$.

41. Li, Y.; Chen, Y.; Podlubny, I. Stability of fractional-order nonlinear dynamic systems: Lyapunov direct method and generalized Mittag-Leffler stability. Comput. Math. Appl. 2010, 59, 1810-1821. [CrossRef]

42. Benchohra, M.; Lazreg, J.E. On stability for nonlinear implicit fractional differential equations. Le Matematiche 2015, 70, 49-61.

43. Ali, Z.; Zada, A.; Shah, K. Ulam stability results for the solutions of nonlinear implicit fractional order differential equations. Hacettepe J. Math. Stat. 2018, 1-18. [CrossRef]

44. Ali, A.; Samet, B.; Shah, K.; Khan, R.A. Existence and stability of solution to a toppled systems of differential equations of non-integer order. Bound. Value Probl. 2017, 2017, 1-16. [CrossRef]

45. Ali, Z.; Zada, A.; Shah, K. Ulam stability to a toppled systems of nonlinear implicit fractional order boundary value problem. Bound. Value Probl. 2018, 2018, 175. [CrossRef]

46. Khan, A.; Shah, K.; Li, Y.; Khan, T.S. Ulam type stability for a coupled systems of boundary value problems of nonlinear fractional differential equations. J. Funct. Spaces, 2017, 2017, 1-8. [CrossRef]

47. Shah, K.; Wang, J.; Khalil, H.; Khan, R.A. Existence and numerical solutions of a coupled system of integral BVP for fractional differential equations. Adv. Differ. Equ. 2018, 2018, 149. [CrossRef]

48. Shah, K.; Tunç, C. Existence theory and stability analysis to a system of boundary value problem. J. Taibah Univ. Sci. 2017, 11, 1330-1342. [CrossRef] 
49. Ali, Z.; Zada, A.; Shah, K. On Ulam's stability for a coupled systems of nonlinear implicit fractional differential equations. Bull. Malays. Math. Sci. Soc. 2018, 1-19. [CrossRef]

50. Rus, I.A. Ulam stabilities of ordinary differential equations in a Banach space. Carpathian J. Math. 2010, 26, 103-107.

(C) 2019 by the authors. Licensee MDPI, Basel, Switzerland. This article is an open access article distributed under the terms and conditions of the Creative Commons Attribution (CC BY) license (http:/ / creativecommons.org/licenses/by/4.0/). 